\title{
Exact Formulation and Analysis for the Bi-Objective Insular Traveling Salesman Problem
}

\author{
Pablo A. Miranda-Gonzalez ${ }^{1, *(\mathbb{D})}$, Javier Maturana-Ross ${ }^{2}$ (D) , Carola A. Blazquez ${ }^{3} \mathbb{D}$ \\ and Guillermo Cabrera-Guerrero ${ }^{4}$ (D) \\ 1 Department of Industrial Engineering, Universidad Católica del Norte, Antofagasta 1270709, Chile \\ 2 School of Industrial Engineering, Pontificia Universidad Católica de Valparaíso, Valparaíso 2362807, Chile; \\ javier.maturana@pucv.cl \\ 3 Department of Engineering Sciences, Universidad Andres Bello, Viña del Mar 2531015, Chile; \\ cblazquez@unab.cl \\ 4 Escuela de Ingeniería Informática, Pontificia Universidad Católica de Valparaíso, Valparaíso 2362807, Chile; \\ guillermo.cabrera@pucv.cl \\ * Correspondence: pablo.miranda@ucn.cl
}

Citation: Miranda-Gonzalez, P.A.; Maturana-Ross, J.; Blazquez, C.A.; Cabrera-Guerrero, G. Exact Formulation and Analysis for the Bi-Objective Insular Traveling Salesman Problem. Mathematics 2021, 9, 2641. https://doi.org/10.3390/ math9212641

Received: 7 September 2021

Accepted: 3 October 2021

Published: 20 October 2021

Publisher's Note: MDPI stays neutral with regard to jurisdictional claims in published maps and institutional affiliations.

Copyright: (c) 2021 by the authors. Licensee MDPI, Basel, Switzerland. This article is an open access article distributed under the terms and conditions of the Creative Commons Attribution (CC BY) license (https:// creativecommons.org/licenses/by/ $4.0 /)$.
Abstract: This paper aims at studying the Bi-Objective Insular Traveling Salesman Problem (BO-InTSP), which searches for a set of efficient, single visit sequences to collect (or distribute) freight from a set of islands. In this problem, the selection of ports (nodes) to be visited at each island, along with the associated port visit sequence, are optimized simultaneously, while the maritime transportation costs and the ground transportation costs inside the islands are minimized with a bi-objective perspective. This approach is employed since these costs are of a conflictive nature. A previous Approximated Formulation of the BO-InTSP relies on aggregating the actual demand locations within each island in a certain number of centroids for computing the ground transportation costs. Conversely, this paper proposes and develops a novel Exact Formulation for the problem based on the actual demand locations, instead of aggregating the demand inside the islands. Additionally, a systematic evaluation approach is developed to compare the two alternative formulations with different levels of demand aggregation inside the islands, considering the bi-objective nature of the problem. The results reveal that the novel Exact Formulation significantly outperforms the previous aggregated approach in terms of the solutions quality and computational resources.

Keywords: insular traveling salesman problem; ground transportation costs; freight collection or distribution; isolated regions; bi-objective optimization; multi-objective analysis

\section{Introduction and Literature Review}

Vehicle Routing Problems (VRPs) have been widely studied for decades to address a great variety of real-world problems that involve freight distribution to (or collection from) a set of locations ([1-6]). In most classical cases, the set of locations or nodes to be visited are previously determined and fixed, denoting the traditional mandatory nature of these problems, as shown in Figure 1a.

Nevertheless, in several situations, the nodes to be served must be selected simultaneously with visit sequences, denoting the selective nature of the visits for these cases (as shown in Figure 1b). Some examples are the Selective VRP, SVRP ([7-10]), Orienteering Problems ([11-17]), and the Generalized VRP, GVRP ([18-27]). With this regard, other related problems belonging to the family of extensive facility location problems, in which the main goal is to determine the topology of the network for serving a set of customers by means of tours, paths, trees, or other types of networked structures have been proposed in the literature ([28-36]). 


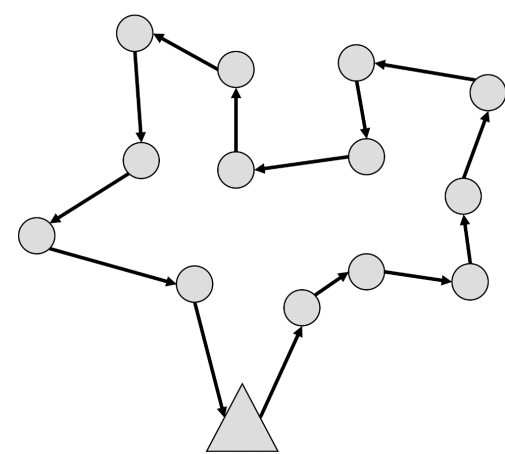

(a)

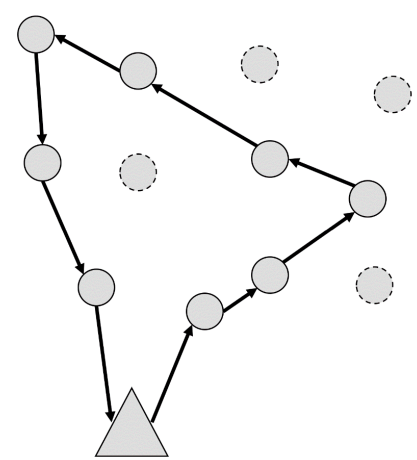

(b)

Figure 1. Solution Examples of Classic and Selective VRP: (a) Classic VRP; (b) Selective VRP.

However, there are a wide variety of applications that involve serving a set of islands or isolated regions (as shown in Figure 2), which cannot be directly addressed by any aforementioned VRPs or related works. Some distinctive features of these applications are the selection of the ports, docks, or locations to visit each island (similar to selective VRPs) jointly with the transportation process and costs inside the islands. Figure 2 shows a simple strategy to transport the freight between demand locations and ports, based on a greedy criterion (i.e., the nearest selected port for each demand location). Note that if the transportation costs inside the islands are insignificant, then the problem becomes the Generalized VRP or the Generalized Traveling Salesman Problem (GTSP). Other advanced transportation processes, such as routing or consolidation inside the islands, can be considered, which may require advanced mathematical models and solution techniques.

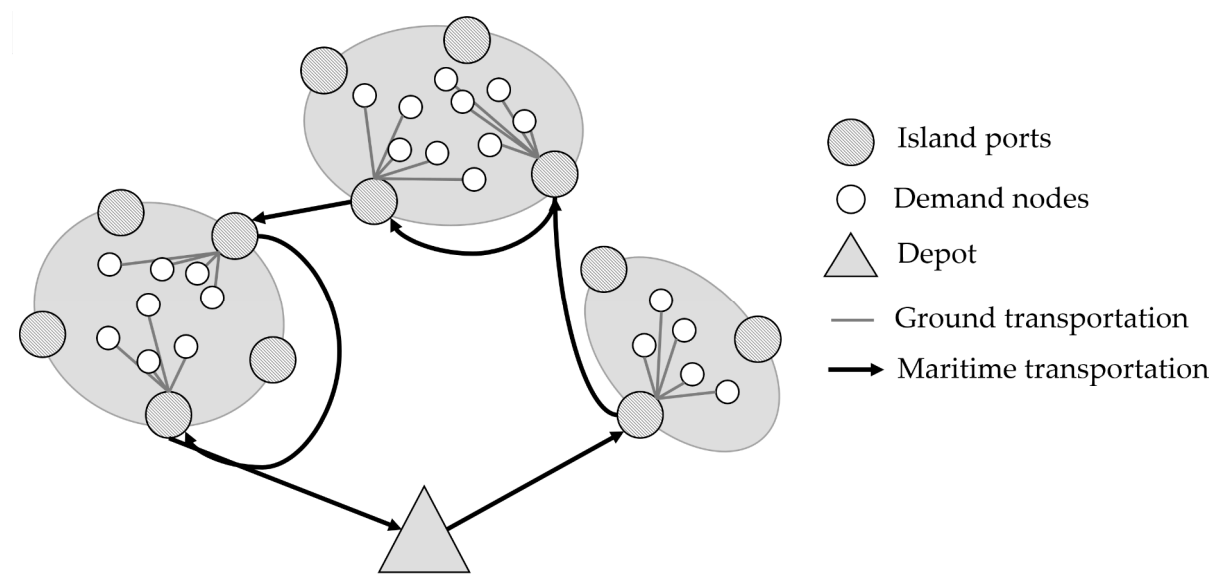

Figure 2. Solution example for insular TSP with a simple, greedy ground transportation process.

Only a few recent studies ([37-39]) have proposed some formulations for this type of problem under different settings and assumptions. These studies introduced formulations as variants of the SVRP and the GVRP. The corresponding class of problems is referred to as the Insular Vehicle Routing Problem (InVRP), including the Insular Traveling Salesman Problem (InTSP) studied by [39] and in this present research.

Motivated by a real case study in an archipelago of Chiloe and Palena in southern Chile, the aforementioned studies predominantly focus on designing a household waste collection system, where a set of small rural islands must be served by one barge to collect household waste. Each island has at least one port that should be selected to operate as a waste collection site. In this problem, the node selection and visit sequence decisions must be optimized simultaneously.

The InVRP modelling structure presents the advantage of being quite appropriate in a variety of applications. Some examples are food deserts in inland regions ([40-45]), and the maintenance of wind farm facilities $([46,47])$. Overall, situations that involve serving 
isolated regions (inland or offshore) for different purposes (e.g., distributing, collection, maintenance, etc.) are suitable applications.

As shown in Figure 2, the ground transportation costs (GTC) and its associated processes inside the islands are relevant drivers for the problem. In this case, each demand location is assigned to the nearest selected port. Based on this ground transportation strategy inside the islands, [39] introduced a first approximation for these costs by explicitly aggregating the actual demand locations with fictitious centroids. Subsequently, based on this approximation, the authors formulated the Bi-Objective Insular Traveling Salesman Problem (BO-InTSP), which consists of minimizing the maritime transportation costs (MTC) incurred by a barge to visit the selected ports, and the GTC incurred inside the islands to transport the freight between users, households, or demand locations, and such selected ports.

It is worth mentioning that the family of clustered VRPs (or TSP) are also related to our research, since they consider the existence of node groups or clusters to be served ([48-50]). However, in these problems, all of the nodes must be visited, as opposed to the BO-InTSP, where visiting all of the nodes is not mandatory.

Representing several real-world problems, multi-objective VRPs have been extensively researched in the literature; see [51-53] for reviews on multi-objective VRPs and related problems. Rather than exact methods, heuristic methods have been mainly applied to multi-objective routing problems for obtaining an approximation to the Exact Pareto Front ([54-57]). Naturally, finding the full Exact Pareto Front for large-size instances is a difficult task in this type of Mixed Integer Programming (MIP), NP-hard problems.

Other problems related to the InVRP and the BO-InTSP are the multi-objective network design problems, in which the topology of the network is designed while optimizing different relevant objectives, such as the total cost, $\mathrm{CO}_{2}$ emissions, and waste generation, among others ([58-60]). Other examples of extensive bi-objective facility locations are the median path problem and the median shortest path problem ([61,62]). Both problems minimize the total distance of the tour and the travel distance from the non-selected nodes to the closest stop on the tour. Furthermore, other variants are the bi-objective ring star problem $([63,64])$, the bi-objective covering tour problem $([61,65])$, and the traveling purchaser problem $([66,67])$.

The main assumptions of the approximation in [39] are: (i) the demands are aggregated and located at a certain number of centroids instead of at the real demand locations, (ii) as input parameters, the total island demand is homogeneously fragmented and assigned to the defined centroids, (iii) each centroid is associated to a single port and vice-versa; thus, if such a port is visited, then the associated centroid demand will be served through this port, and (iv) if a port is not visited (i.e., a non-selected port), then the demand of its associated centroid will be homogeneously split among the other selected ports of the island.

In some cases, when real information is not completely available or computational issues arise, this aggregated approximation may be a reasonable approach. However, there is a lack of evidence about the quality of this approximation, and, moreover, there is no systematic methodology to assess its quality. Notice that if an inappropriate GTC approximation is employed, then an incorrect port selection would be obtained in terms of the quantity and location, thus yielding solutions with an inadequate trade-off between MTC and GTC. Thus, a thorough analysis of the solutions obtained by the Approximated Model and the Exact Formulation is worth studying.

This paper proposes a novel Exact Formulation for the BO-InTSP based on the actual demand locations inside the islands, assuming that each user or inhabitant would prefer the nearest operating port (i.e., node), instead of aggregating demand locations at a set of fictitious centroids, as in [39]. Subsequently, this research proposes and develops a systematic evaluation approach to compare the sets of non-dominated points obtained with the two bi-objective formulations using the same exact algorithm.

It is worth highlighting that the proposed evaluation approach substantially differs from traditional multi-objective approaches, which usually compare the sets of non- 
dominated points generated by different approximated algorithms (i.e., heuristic) for a single problem or model formulation. Thus, this research contributes to an enhanced analysis and comparison among models with different accuracy or aggregation levels. The proposed approach might be particularly important when trying to balance the effort needed to solve a problem either through an Exact Formulation or through an Approximated Model, as in this research. Furthermore, the proposed strategy employed to compare different models may be extended to models with more than two objectives (multi-objective problems).

In multi-objective optimization, several performance indicators exist to measure the quality of a given set of non-dominated points ([68-71]). Some examples of these indicators are the hypervolume index (or dominated area for the 2-dimension case), uniformity index, covering index, or simply the obtained number of non-dominated points. In general, finding a good approximation to the set of non-dominated points would be equivalent to: (i) maximize the number of obtained non-dominated points, (ii) maximize the associated dominated area, (iii) minimize the distance between each pair of non-dominated points, and (iv) maximize the range covered by the set of non-dominated points for each objective function. As can be observed, the problem of finding a good quality set of non-dominated points is a multi-objective problem itself. Usually, all these quality indicators are employed to compare the performance of different multi-objective heuristic algorithms based on the sets of (approximated) non-dominated points found by each algorithm. It is clear that this kind of performance indicators are not needed if an algorithm is able to provide the actual set of non-dominated points.

Thus, this paper aims at evaluating and comparing the set of non-dominated points obtained by using the Approximated Model with respect to the set of non-dominated points obtained by the novel Exact Formulation. This comparison involves the hypervolume index among other natural performance indicators. Furthermore, note that the actual set of non-dominated points obtained by the Approximated Model is indeed an approximation to the actual set of non-dominated points of the Exact Formulation.

Summarizing, this paper proposes an Exact Formulation for the BO-InTSP, and compares the results obtained with this formulation to those associated with the aggregatedbased mathematical formulation (i.e., Approximated Model) in [39]. The comparison is focused on the set of non-dominated points obtained when employing each formulation.

Three types of centroids were tested for the aggregated formulation: (i) manually defined centroids, (ii) geometric centroids based on the shape of the islands, and (iii) centre-of-mass obtained by averaging the coordinates of the non-aggregated demand locations. Therefore, the comparison shows the effect of using different procedures to determine the centroid locations. The Pareto Front for each formulation is obtained using the AUGMECON2 method described in [72,73].

The remainder of this paper is organized as follows. Section 2 presents the problem description, the previous Approximated Model, and the proposed novel Exact Formulation. Section 3 introduces the computational application along with a description of methodological foundations required for its implementation and analysis. Section 4 presents and analyzes the main results from the computational applications. Finally, Section 5 concludes the main findings of this work.

\section{Problem Description and Formulations}

\subsection{General Problem Description}

The BO-InTSP aims at determining a set of efficient, single sequences to visit a collection of islands with a single barge, while minimizing both the MTC and GTC based on a bi-objective approach. In this problem, the barge must collect all of the freight in a single period (e.g., a day or a week), and it is assumed that the barge has a sufficient capacity for collecting all of the freight. The decisions involved in this problem are the port or dock selection at each island along with the respective visit sequence. Finally, a single depot or transfer station is considered as the start and end of the barge route. 
Each island has one or more available ports that may be potentially employed as a collection site, as indicated in Figure 3. Accordingly, the model should optimize the collection site selection and the visit sequence of the selected ports, ensuring that each island is visited at least in one port. Note that all selected nodes of an island are not necessarily visited consecutively prior to visiting other islands. In the BO-InTSP, the total MTC of the barge for visiting all selected ports, and the total GTC incurred by its inhabitants to carry the freight to the selected ports at each island, must be minimized. Figure 3 presents two examples in which a single visit sequence includes only one port per island (Figure 3a) and all island ports (Figure 3b).

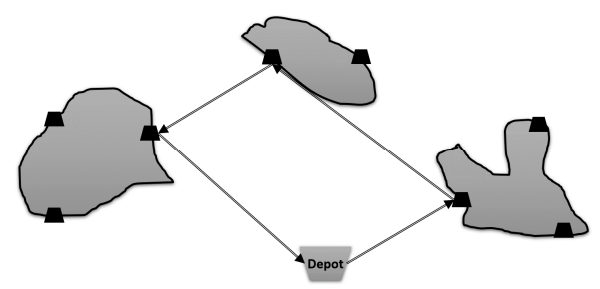

(a)

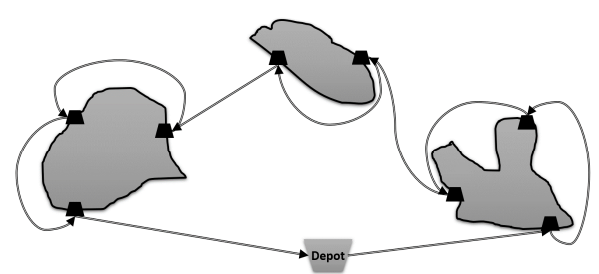

(b)

Figure 3. Examples of feasible solutions for the Insular Traveling Salesman Problem (InTSP): (a) solution example visiting one port per island; (b) solution example visiting all ports.

In the studied waste collection case, the freight inside the islands is generated by a set of rural households, and the inhabitants must transport their freight to the ports by employing modest transportation (e.g., walking, cow, tumbril, horse, etc.). In this scenario, the GTC inside the islands is incurred by inhabitants, whereas the MTC is incurred by local authorities or a logistic provider to serve the islands. In other words, these costs are incurred by different agents or stakeholders. If only the GTC is minimized, then the problem leads to a solution where all of the ports are visited, as in Figure 3a, since the freight of each centroid is picked up using its nearest port. On the contrary, if only the MTC is minimized, then the problem may lead to a solution where only one port is visited for each island, as in Figure 3b. Therefore, the GTC and MTC objectives are in conflict; therefore, a bi-objective approach must be adopted. Thus, the set of efficient solutions that jointly minimize both the MTC and the GTC should be found. Each efficient solution comprises both the selection of the ports that must be visited and the visit sequence of such ports. Considering that the Selective Traveling Salesman Problem (STSP) and the GTSP are both NP-hard problems $([7,27])$, and that they are particular cases of the InTSP (if all of the nodes or ports conform a single big island, then the InTSP becomes a STSP; furthermore, if the ground transportation cost is zero, then the InTSP becomes a GTSP), then InTSP is NP-hard as well.

The following Sections 2.2 and 2.3 describe the previous Approximated Model and the proposed Exact Formulation for the studied problem, respectively.

\subsection{Approximated Model Based on Demand Aggregation}

In [39], the authors proposed an approximated approach for computing the GTC. In this approximation, it is assumed that each island is fictitiously segmented into a certain number of zones. Each zone is associated with the nearest port and vice-versa. These fictitious zones are defined so that if all ports of an island are selected, then the freight generated at each zone will be fully collected through its associated port. Furthermore, each zone is represented by a fictitious centroid that concentrates the total freight of the zone.

Hereafter, the location of each centroid and its associated freight must be computed as model parameters. In [39], a manual procedure is employed to locate the centroid for each zone, and the total freight of the island is homogeneously split among the centroids. These assumptions are motivated by a complete absence of information related to freight generation locations within each island. 
An additional assumption is employed for splitting the freight of each centroid among the selected ports on the island. This assumption is relevant only if the port associated to this centroid is not selected and its freight should be collected through other selected ports. In this case, the Approximated Model assumes a linear modelling structure, where the freight located at a centroid is homogeneously split among the other selected ports. Otherwise, if the associated port is selected, then the centroid demand will be completely collected through this port.

The sets, parameters, and decision variables of the problem are the following:

$H$ : Set of islands to be served by a barge;

$\mathrm{N}$ : Set of nodes, including only the ports at the islands;

$N_{0}$ : Set of nodes, including ports and the depot $i_{0}$;

$\Omega_{h}$ : Set of nodes at each island $h$;

$\Psi_{h}$ : Set of all possible combinations of ports for island $h$;

$K_{h s}$ : Set of nodes belonging to island $h$ that are selected as collection sites under combination $s \in \Psi_{h}$;

$\delta_{h}$ : Number of nodes at each island $h$;

$M C_{i j}$ : MTC from node $i$ to node $j$;

$Q_{h}$ : Freight volume to be collected from each island $h$;

$G C_{h s}$ : Total GTC at island $h$, if the port combination $s$ is selected. This cost parameter represents the total GTC incurred by the island inhabitants if port combination $s$ is selected;

$Z i$ : Binary variable indicating if node $i$ is selected to be visited;

$Y_{i j}$ : Binary variable indicating if node $j$ is visited immediately after node $i$;

$X_{h s}$ : Binary variable indicating if island $h$ is visited using combination $s$;

$F_{i j}$ : Fictitious flows from node $i$ to node $j$.

Accordingly, the Approximated Model is formulated as (1)-(13). Expressions (1) and (2) correspond to the MTC and GTC objective functions, respectively. Constraints (3) ensure the exact selection of a single port scenario $s$ for each island $h$. Constraints (4) and (5) are logical relationships between the decision variables $Z$ and $X$, ensuring that if a combination of port $\mathrm{s}$ is selected for each island $\mathrm{h}\left(X_{h s}=1\right)$, only the selection variables for the associated ports are activated $\left(Z_{i}=1\right.$, for each $\left.i \in K_{h s}\right)$. Constraints (6) and (7) ensure that, for each selected port (i.e., $Z_{i}=1$ ), the barge must enter and exit exactly once, respectively. Constraints (8)-(11) are the sub-tour elimination constraints ([74]). Finally, Constraints (12) and (13) are of domain.

$$
\begin{gathered}
\text { Min } \sum_{i, j \in N_{0}, j \neq i} M C_{i j} \cdot Y_{i j} \\
\text { Min } \sum_{h \in H \in H \in \Psi_{h}} G C_{h s} \cdot X_{h s} \\
\text { subject to }: \\
\sum_{s \in E_{h}} X_{h s}=1 \quad \forall h \in H \\
X_{h s} \leq Z_{i} \quad \forall h \in H, s \in E_{h}, i \in \Omega_{h} / i \in K_{h s} \\
X_{h s} \leq 1-Z_{i} \quad \forall h \in H, s \in E_{h}, i \in \Omega_{h} / i \notin K_{h s} \\
\sum_{j \in N: i \neq j} Y_{i j}=Z_{i} \quad \forall i \in N \\
\sum_{j \in N: j \neq i} Y_{j i}=Z_{i} \quad \forall i \in N \\
\sum_{j \in N} F_{i_{0} j}=\sum_{j \in N} Z_{j}
\end{gathered}
$$




$$
\begin{gathered}
\sum_{j \in N_{0} / i \neq j} F_{i j}=\sum_{j \in N_{0} / i \neq j} F_{j i}+Z_{i} \quad \forall i \in N \\
\sum_{j \in N} F_{j i_{0}}=0 \\
F_{i j} \leq|N| \cdot Y_{i j} \quad \forall i, j \in N_{0}, i \neq j \\
F_{i j} \geq 0 \quad \forall i, j \in N_{0}, i \neq j \\
Z, Y_{i j}, X_{h s} \in\{0,1\} \quad \forall i, j \in N, \forall h \in H, \forall s \in E_{h}
\end{gathered}
$$

\subsection{An Exact Formulation for the BO-InTSP}

The proposed novel exact mathematical formulation for the BO-InTSP considers a disaggregated scheme, relying on the knowledge of the real demand locations (e.g., households, restaurants, or hospitals) and their respective demand values. In this model, island inhabitants must travel to their nearest selected port for transporting their freight. Thus, when capacity constraints at the ports are not relevant, the model will allocate each demand location to its nearest selected port, as shown in Figure 4. It can be shown that any efficient solution is equivalent to a greedy assignment scheme that all inhabitants may follow. Note that this figure presents an example of a part of a complete feasible solution (ground transportation component).

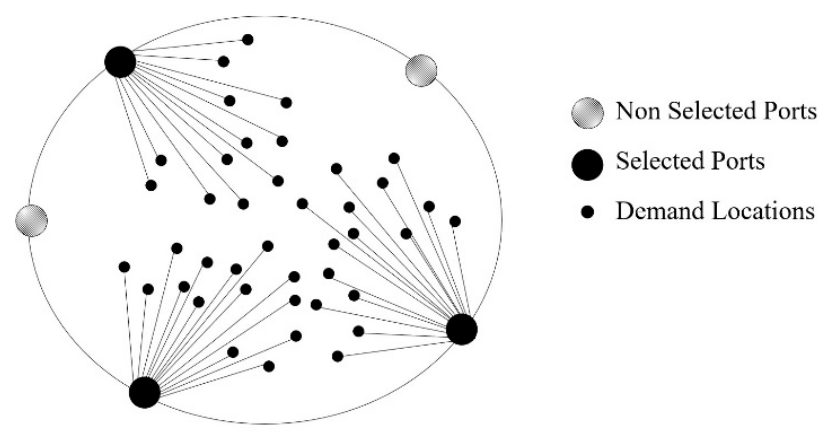

Figure 4. Example of demand location assignments to the selected ports inside the islands.

Under this alternative disaggregated scheme, the total GTC for every island is explicitly computed based on the actual allocation of the demands to the selected ports. The proposed model contains additional variables compared to the previous Approximated Model but provides a better problem representation.

The Exact Formulation considers the definition of the following additional parameters and decision variables:

$M_{h}$ : Set of demand locations belonging to each island $h$;

$d_{i l}$ : Distance between port $i$ and demand location $l$;

$q_{l}$ : Demand of location $l$;

$\phi_{i l}$ : Binary variable indicating if demand location $l$ is allocated to node $i$.

Due to the absence of freight generation information for each specific demand location, it is assumed that the total demand of an island is homogeneously split among all demand locations, as $q_{l}=Q_{h} /\left|M_{h}\right|, \forall h \in H, \forall l \in M_{h}$. Notice that this assumption does not affect the structure of the Exact Formulation, since $q_{l}$ is only a parameter of the model that may be computed following any other information or assumption.

Consequently, the novel Exact Formulation of the BO-InTSP is as (14) to (27). Expressions (14) and (15) correspond to the MTC and GTC objective functions, respectively. MTC is the same as the Approximated Model, whereas GTC corresponds to a new Exact Formulation based on demand location assignment decisions. Constraints (16)-(18) are new constraints that allow for computing GTC in an exact manner. Constraints (16) ensure that at least one port is selected for each island. Constraints (17) guarantee that each demand location is assigned to a single port, and restrictions (18) allow for assignment decisions $\left(\phi_{i j}\right)$ 
only involving selected ports $\left(Z_{i}=1\right)$. Expressions (19)-(24) are routing-related constraints, and are equivalent to Equations (6)-(11). Finally, Constraints (25)-(27) are of domain.

$$
\begin{aligned}
& \operatorname{Min} \sum_{i, j \in N_{0}, j \neq i} M C_{i j} \cdot Y_{i j} \\
& \operatorname{Min} \sum_{h \in H} \sum_{i \in \Omega_{h}} \sum_{l \in M_{h}} d_{i l} \cdot q_{l} \cdot \phi_{i l} \\
& \text { subject to : } \\
& \sum_{i \in \Omega_{h}} Z_{i} \geq 1 \quad\left(Z_{i_{0}}=1\right) \quad \forall h \in H \\
& \sum_{i \in \Omega_{h}} \phi_{i l}=1 \quad \forall h \in H, l \in M_{h} \\
& \phi_{i l} \leq Z_{i} \quad \forall h \in H, l \in M_{h}, i \in \Omega_{h} \\
& \sum_{j \in N: i \neq j} Y_{i j}=Z_{i} \quad \forall i \in N \\
& \sum_{j \in N: j \neq i} Y_{j i}=Z_{i} \quad \forall i \in N \\
& \sum_{j \in N} F_{i_{0} j}=\sum_{j \in N} Z_{j} \\
& \sum_{j \in N_{0} / i \neq j} F_{i j}=\sum_{j \in N_{0} / i \neq j} F_{j i}+Z_{i} \quad \forall i \in N \\
& \sum_{j \in N} F_{j i_{0}}=0 \\
& F_{i j} \leq|N| \cdot Y_{i j} \quad \forall i, j \in N_{0}, i \neq j \\
& F_{i j} \geq \quad \forall i, j \in N_{0}, i \neq j \\
& Z_{i}, Y_{i j} \in\{0,1\} \quad \forall i, j \in N, \forall h \in H, \forall s \in E_{h} \\
& \phi_{i l} \in\{0,1\} \quad \forall h \in H, l \in M_{h}, i \in \Omega_{h}
\end{aligned}
$$

\section{Computational Issues and Comparison Methodology}

This section details the computational experience and methodological foundations of the proposed comparison approach. The goal is to compare the results obtained with both the Approximated Model ([39]) and the Exact Formulation proposed in this research (Section 2.3).

The two formulations are solved using the AUGMECON2 method described in [72]. This method guarantees obtaining the Exact Pareto Front for multi-objective mixed integer programming problems whose objective functions involve only integer variables (which are satisfied in both formulations). Additionally, possessing the nadir point is required, which, in this implementation, is obtained at the beginning of the algorithm.

The AUGMECON2 algorithm works similarly to the well-known epsilon constraints algorithm, in which a mono-objective reformulation of the original multi-objective problem is solved iteratively by optimizing only one of the objective functions, and the remaining functions are instead handled as model constraints. The AUGMECON2 algorithm performs an accelerated update of the right-hand side parameters of the model constraints associated with the objective functions. For more details, see [72,73]. The algorithm was implemented using AMPL as a programming language, CPLEX 12.8 as the optimization package, and a computer with an Intel i7-4770 processor @ $3.40 \mathrm{GHz}$ with 8 GB RAM, where the number of threads is set to one. 
It is worth highlighting that, depending on the approach employed to obtain the Pareto Front of a bi-objective problem, different results may be produced. For example, if heuristic approaches are employed, only approximated Pareto Fronts would be obtained, and there would not be a guarantee for obtaining optimal solutions and the Exact Pareto Fronts. Thus, any comparison would not be completely definitive or conclusive. However, in this research, we aim to compare the exact Pareto Fronts obtained for both formulations (the exact and the approximated models) in order to gain more conclusive insights. Thus, a state-of-the-art, exact solution approach for multi-objective MIP problems is employed; in this case, the AUGMECON2 algorithm. Nonetheless, it is possible and advisable to develop or apply advanced solution approaches, particularly for larger instances, such as Multi Objective Evolutionary Algorithms based on Decomposition (MOEA/D), Non-Sorting Genetic Algorithms II (NSGA-II), and Pareto Local Search, including all of its variations and improvements ([75-80]).

\subsection{Test Instances and Experiment Design}

This study considers a variety of instances that are based on two original instances presented in [39]: one real and one fictitious. The original real instance contains 21 islands from southern Chile, from which, 11 islands have 1 port, 8 islands have 2 ports, and 2 islands have 3 ports. The original fictitious instance comprises 20 synthetic circular islands, where 8 islands contain 2 ports, 8 islands contain 3 ports, and 4 islands contain 4 ports. A first set of real instances are generated by randomly selecting islands from the original real instance with 21 islands. Similarly, a second set of fictitious instances are generated from the original synthetic instance with 20 circular islands.

Although larger instances may be solved to optimality for the single objective TSP in a reasonable time, solving its bi-objective version is more difficult from a computational point of view. Therefore, this research considers small and medium-sized instances. Furthermore, given that this study aims at solving the two formulations at optimality, the resolution of larger instances is prohibitive. In this case, the use of non-exact algorithms or heuristics is recommended, which is out of the scope of this study.

The two original instances were built considering an aggregated GTC, in which the demands were modelled by employing aggregated centroids at each island. Conversely, the proposed disaggregated Exact Formulation relies on the direct GTC (i.e., Euclidean distances) between the disaggregated demand locations and the island ports. Accordingly, disaggregated demand locations inside the islands are randomly generated in this study, since real demand locations are not available.

For solving the Approximated Model, three alternative centroid generation approaches, namely Manual Centroids, Geometric Centroids, and Centre-of-Mass, are employed for each instance. Only Manual and Centre-of-Mass centroids are employed for the real instances. For solving the Exact Formulation, disaggregated demand locations were created in a random manner independently for the real and the fictitious instances, yielding between 22 and 50 nodes per island. Homogeneous demands are considered for all generated demand locations. Furthermore, the Centre-of-Mass centroids employed for the Approximated Model are computed using the coordinates of these disaggregated demand locations.

The computational experience is divided into two parts. The first experiment (Part I) aims at showing and analyzing the conceptual and structural differences between the set of non-dominated points obtained with both the Approximated and the Exact Formulations considering only small instances. For this analysis, one real and three fictitious instances are generated based on the two aforementioned original instances. These are defined as Real-0820, Fict-0660, Fict-0064, and Fict-0004, where the digits of each instance name (real or fictitious) indicate the number of islands with 1, 2, 3, or 4 ports, respectively. For example, Real-0820 denotes 0 islands with 1 port, 8 islands with 2 ports, 2 islands with 3 ports, and 0 islands with 4 ports. 
The second experiment (Part II) aims at evaluating the aggregated behavior and the computational performance of the two formulations. In this case, only Centre-of-Mass is employed for generating centroids, given the results of the first computational experience discussed in Section 4.1. This experiment focuses on solving 10 real instances with 18 islands that are randomly selected from the original 21-island real instance, which comprise 8 islands with 1 port, 8 islands with 2 ports, and 2 islands with 3 ports. Additionally, 10 fictitious instances are considered containing 17 islands that are randomly selected from the original 20-island fictitious instance, where each instance comprises 7 islands with 2 ports, 7 islands with 3 ports, and 3 islands with 4 ports. Following a similar notation associated with the first part of the experiment, the large instances are named as Real-wxyz$\mathrm{n}$ and Fict-wxyz-n, where $\mathrm{w}, \mathrm{x}, \mathrm{y}$, and $\mathrm{z}$ define the number of islands with 1, 2, 3 and 4 ports, respectively, and the additional index $\mathrm{n}$ defines a correlative identification number for each instance, ranging from 01 to 10 . Table 1 summarizes the instances considered in this study.

Table 1. Experiments and instance summary.

\begin{tabular}{|c|c|c|c|c|c|c|c|c|}
\hline Experiment & $\begin{array}{l}\text { Instance } \\
\text { Name }\end{array}$ & $\begin{array}{l}\text { Type of } \\
\text { Islands }\end{array}$ & $\begin{array}{l}\text { \# of Nodes } \\
\text { (Depot } \\
\text { Included) }\end{array}$ & $\begin{array}{c}\text { \# of } \\
\text { Islands }\end{array}$ & $\begin{array}{c}\text { \# Islands } \\
\text { with } \\
1 \text { Port }\end{array}$ & $\begin{array}{c}\text { \# Islands } \\
\text { with } \\
2 \text { Ports }\end{array}$ & $\begin{array}{c}\text { \# Islands } \\
\text { with } \\
3 \text { Ports }\end{array}$ & $\begin{array}{c}\text { \# Islands } \\
\text { with } \\
4 \text { Ports }\end{array}$ \\
\hline \multirow{4}{*}{ Part I } & Real-0820 & Real & 23 & 10 & 0 & 8 & 2 & 0 \\
\hline & Fict-0660 & \multirow{3}{*}{ Fictitious } & 31 & 12 & 0 & 6 & 6 & 0 \\
\hline & Fict-0064 & & 35 & 10 & 0 & 0 & 6 & 4 \\
\hline & Fict-0004 & & 17 & 4 & 0 & 0 & 0 & 4 \\
\hline \multirow{20}{*}{ Part II } & Real-8820-01 & \multirow{10}{*}{ Real } & 31 & 18 & 8 & 8 & 2 & 0 \\
\hline & Real-8820-02 & & 31 & 18 & 8 & 8 & 2 & 0 \\
\hline & Real-8820-03 & & 31 & 18 & 8 & 8 & 2 & 0 \\
\hline & Real-8820-04 & & 31 & 18 & 8 & 8 & 2 & 0 \\
\hline & Real-8820-05 & & 31 & 18 & 8 & 8 & 2 & 0 \\
\hline & Real-8820-06 & & 31 & 18 & 8 & 8 & 2 & 0 \\
\hline & Real-8820-07 & & 31 & 18 & 8 & 8 & 2 & 0 \\
\hline & Real-8820-08 & & 31 & 18 & 8 & 8 & 2 & 0 \\
\hline & Real-8820-09 & & 31 & 18 & 8 & 8 & 2 & 0 \\
\hline & Real-8820-10 & & 31 & 18 & 8 & 8 & 2 & 0 \\
\hline & Fict-0774-01 & \multirow{10}{*}{ Fictitious } & 52 & 18 & 0 & 7 & 7 & 4 \\
\hline & Fict-0774-02 & & 52 & 18 & 0 & 7 & 7 & 4 \\
\hline & Fict-0774-03 & & 52 & 18 & 0 & 7 & 7 & 4 \\
\hline & Fict-0774-04 & & 52 & 18 & 0 & 7 & 7 & 4 \\
\hline & Fict-0774-05 & & 52 & 18 & 0 & 7 & 7 & 4 \\
\hline & Fict-0774-06 & & 52 & 18 & 0 & 7 & 7 & 4 \\
\hline & Fict-0774-07 & & 52 & 18 & 0 & 7 & 7 & 4 \\
\hline & Fict-0774-08 & & 52 & 18 & 0 & 7 & 7 & 4 \\
\hline & Fict-0774-09 & & 52 & 18 & 0 & 7 & 7 & 4 \\
\hline & Fict-0774-10 & & 52 & 18 & 0 & 7 & 7 & 4 \\
\hline
\end{tabular}

\subsection{Multi-Objective Comparison Approach}

Following [81,82], the concept of the dominated area, DA (also known as the hypervolume or $S$ metric), is employed to compare the set of non-dominated points obtained with the two formulations, as illustrated with the examples in Figure 5. This figure presents different Pareto Fronts in the objective space, which comprises the values of the objective functions for feasible solutions. The index DA represents the area inside the rectangle limited by $\left(f_{1}^{\min }, f_{2}^{\min }\right),\left(f_{1}^{\max }, f_{2}^{\min }\right),\left(f_{1}^{\min }, f_{2}^{\max }\right)$, and $\left(f_{1}^{\max }, f_{2}^{\max }\right)$ that is weakly dominated by a set of non-dominated points, as shown with the grey area in Figure 5a, where the reference points $\left(f_{1}^{\min }, f_{2}^{\min }\right)$ and $\left(f_{1}^{\max }, f_{2}^{\max }\right)$ are the ideal and the anti-ideal points, respectively. As may be expected, more accurate and complete sets of non-dominated points yield larger values of the DA. 


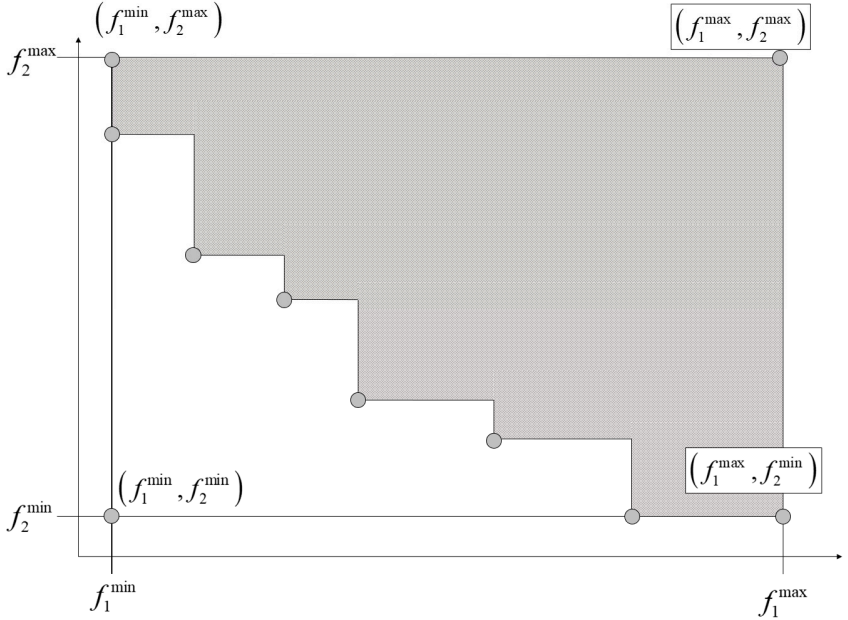

(a)

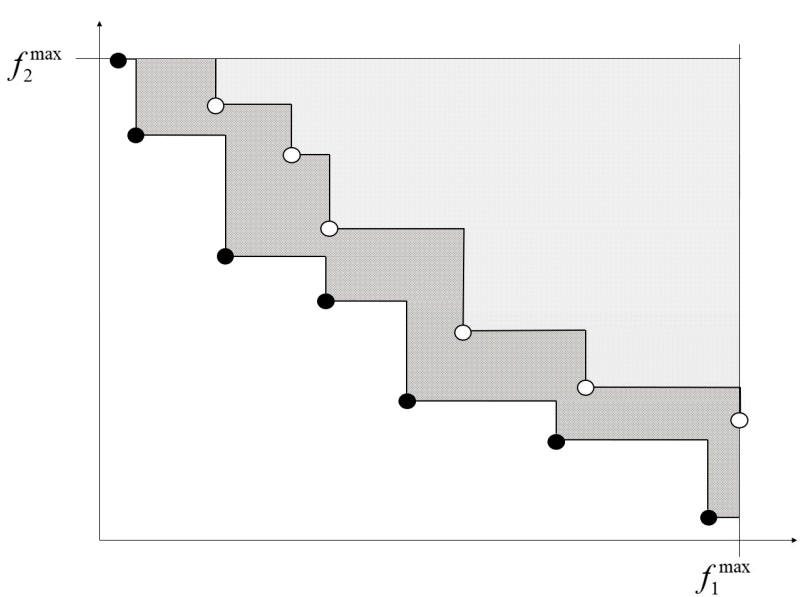

(b)

Figure 5. Example of hypervolume or dominated area (DA): (a) DA of a single Pareto Front; (b) DA comparison between two Pareto Fronts.

Normally, sets with more non-dominated points that are uniformly distributed along the objective space, and closer to the ideal point, yield higher DA values. In Figure 5b, the DAs of two sets are partially overlapped, where the Pareto Front with black dots presents a higher DA value than the Pareto Front with white dots. Particularly, the true set of non-dominated points provides the maximum feasible value for the DA index. Thus, a goal in our study is to compute the hypervolume for two Pareto Fronts: one Pareto Front is obtained with the Approximated Model and another Pareto Front is obtained with the Exact Formulation.

In order to perform a proper comparison between the solutions obtained with the two formulations, the actual set of non-dominated points obtained with the Approximated Model must be projected into the objective space of the Exact Formulation. The projection of points into the objective space of the Exact Formulation consists of evaluating the solutions obtained with the Approximated Model with the objective function of the Exact Model. Consequently, these projected points represent an approximation to the true Pareto Front of the Exact Formulation. Let this projected set be the Approximated Pareto Front, and the true set of non-dominated points obtained by the Exact Formulation be the Exact Pareto Front. Naturally, it is expected that some points belonging to the Approximated Pareto Front are dominated by some points belonging to the Exact Pareto Front, whereas other points may coincide with points of the Exact Pareto Front. An Approximated Pareto Front can be considered a good approximation to the Exact Pareto Front if it contains points that are close to the Exact Pareto Front. Furthermore, for a very good approximation, a large share of its points coincides with some points of the Exact Pareto Front.

In addition to the DA index, a variety of error measurements are computed to evaluate the quality of the points obtained with the Approximated Model after projecting them into the objective space associated with the Exact Formulation. These error measurements are computed by comparing each point of the Approximated Pareto Front to all points from the Exact Pareto Front that dominate this point, as shown in Figure 6. If a point of the Approximated Pareto Front coincides to a point belonging to the Exact Pareto Front, then its associated error is zero. Naturally, the smaller the error, the better the Approximated Pareto Front, and, therefore, the Approximated Model. 


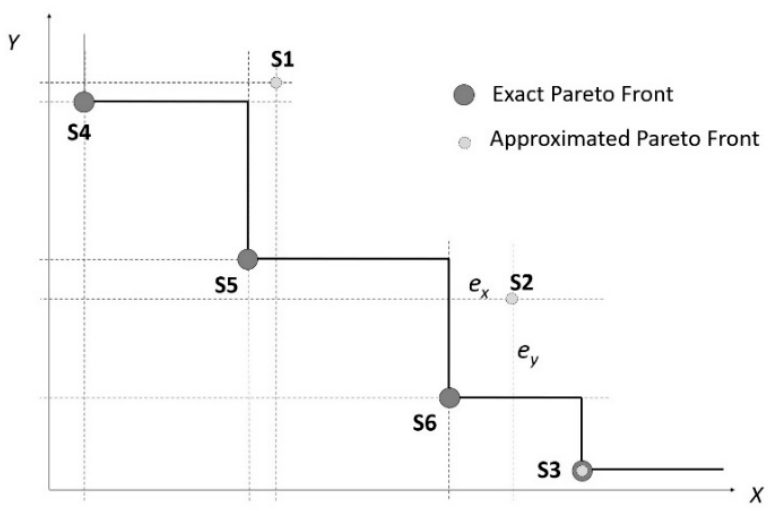

Figure 6. Errors for a dominated solution with respect to non-dominated points.

In the example of Figure 6, points S1, S2, and S3 are part of an Approximated Pareto Front, whereas S3, S4, S5, and S6 belong to the Exact Pareto Front. Notice that a point belonging to the Exact Pareto Front may also be obtained through the Approximated Model, as S3, whose error is zero. On the contrary, some points in the Approximated Pareto Front may be dominated by some points belonging to the Exact Pareto Front. In the example of Figure 6, S2 is dominated by S6, and S1 is dominated by S4 and S5. In this case, when comparing S2 with $\mathrm{S6}$, a relative error $e$ is defined for each objective component, as shown in Equation (28). In this study, $x$ and $y$ refer to the MTC and the GTC, respectively.

$$
e_{x}^{S 6, S 2}=\frac{x_{S 2}-x_{S 6}}{x_{S 6}}, \quad e_{y}^{S 6, S 2}=\frac{y_{S 2}-y_{S 6}}{y_{S 6}}
$$

Based on Expression (3), and considering that $P$ is the Exact Pareto Front and $\widetilde{P}$ is the Approximated Pareto Front, two types of errors are defined: the maximum and Euclidean errors for each point $k \in \widetilde{P}$, with respect to each point $j \in P$ that dominates point $k$. Both expressions are associated with alternative norms of a vector, where norm $\infty$ is associated with the maximum error and norm 2 is associated with the Euclidean error. These errors are shown in Expression (29), where $j<k$ indicates that point $j$ dominates point $k$.

$$
e_{\infty}^{j, k}=\max \left\{e_{x}^{j, k} ; e_{y}^{j, k}\right\} ; e_{2}^{j, k}=\sqrt{\left(e_{x}^{j, k}\right)^{2}+\left(e_{y}^{j, k}\right)^{2}} \forall j \in P, \forall k \in \widetilde{P} / k \succ j
$$

Finally, considering that more than one point from the Exact Pareto Front may dominate a point from the Approximated Pareto Front, a combined error is computed by considering the minimum value for each type of norm (norm $\infty$ and norm 2 ), as indicated in Expression (30) and (31).

$$
\begin{aligned}
& \text { Min_e } e_{\infty}^{k}=\min _{j \in P / k \succ j}\left\{e_{\infty}^{j, k}\right\} \\
& \text { Min_e } e_{2}^{k}=\min _{j \in P / k \succ j}\left\{e_{2}^{j, k}\right\}
\end{aligned}
$$

\section{Results and Discussion}

\subsection{Results for Small Instances}

In this section, the results associated with both the approximated and the exact formulations are presented and discussed for one real and three synthetic instances, as described in Section 3.1. As previously mentioned, this comparison is performed by contrasting the set of non-dominated points obtained by the Approximated Model with the set of non-dominated points obtained with the Exact Formulation. In addition, since GTC is not computed in an exact manner within the Approximated Model, this cost must be recomputed for each obtained non-dominated point prior to performing an appropriate comparison. Subsequently, the comparison assumes the following definitions: 
NDP*: Exact Pareto Front (set of non-dominated points obtained with the Exact Formulation);

NDP-0: Exact set of non-dominated points obtained with the Approximated Model;

DP-1: Set of points in NDP-0 that are dominated by points in NDP* after GTC is recomputed;

NDP-1: Set of points in NDP-0 that are actually non-dominated after GTC is exactly computed. This set is also referred to as the Approximated Pareto Front;

DP-2: Set of points in NDP-1 that do not belong to NDP*;

NDP-2: Set of points in NDP-1 that belong to NDP*.

Figure 7 shows the set of non-dominated points obtained with the Approximated Model (NDP-0) for the instance Fict-0660 using Manual Centroids. The set of non-dominated points with the approximated GTC is shown with grey dots, whereas the points associated with the same solutions with an exact GTC computation is denoted by black dots and grey triangles. Notice that the MTC remains constant for each point, whereas the GTC vertically varies. For this instance, the approximated GTC is always lower than the exact GTC for each point, as shown in Figure 7. As a consequence, once the exact GTC is computed, some points are dominated by others belonging to the same original set, and thus, they must be removed. In other words, the solutions associated with these points are not efficient for the Exact Formulation. The removed points are represented by grey triangles in Figure 7 (DP-1), whereas the black dots denote those points that remain as non-dominated (NDP-1).

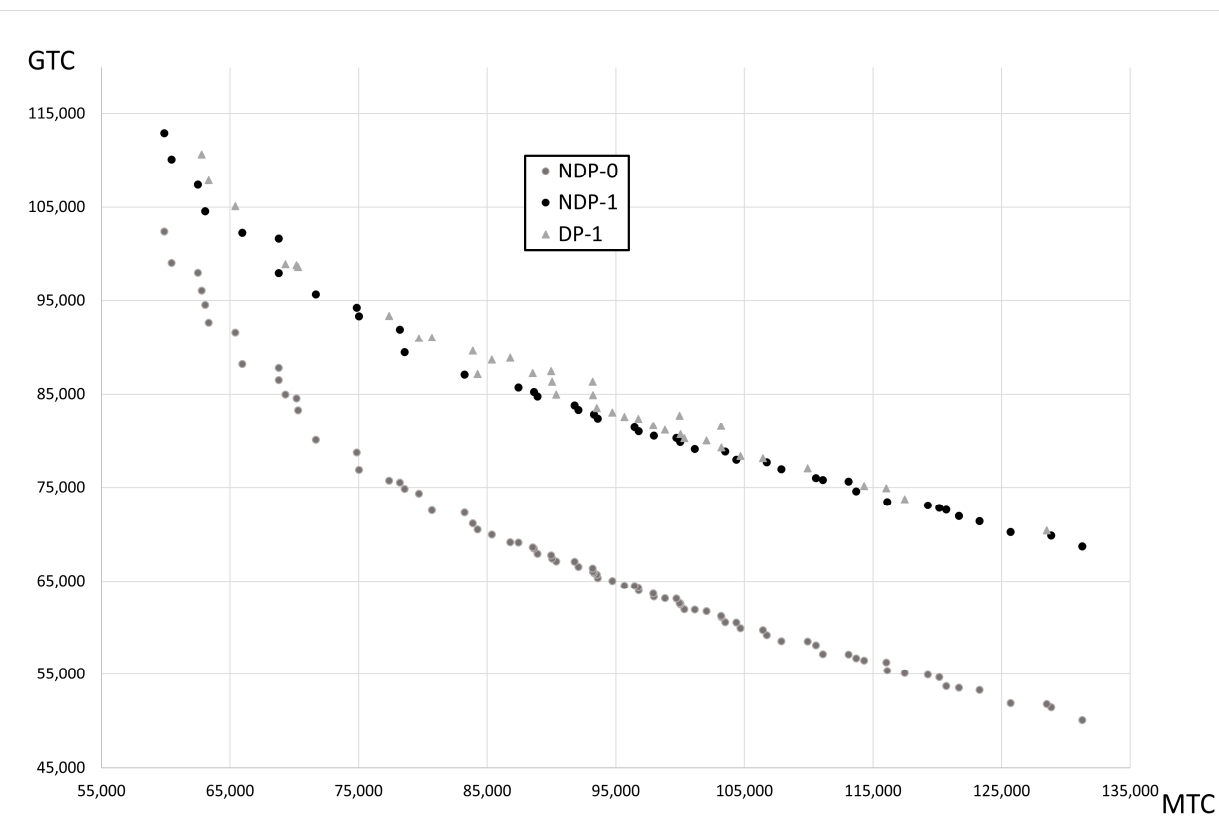

Figure 7. Points associated with efficient solutions to the Aggregated Formulation using Manual Centroids for instance Fict-0660.

For this instance, 81 points (grey dots) are originally obtained for the approximated GTC using Manual Centroids (NDP-0), whereas only 43 points (black dots) remain as non-dominated points after the exact GTC is computed (NDP-1). As expected, only a part of NDP-1 belongs to the exact set of non-dominated points (NDP*), which may be observed once the two sets are contrasted (see Figure 8). In this example, NDP* comprises 127 points, and only 17 points from NDP-1 belong to NDP* (i.e., NDP-2). The remaining 26 points (DP-2) are dominated by other points in NDP*. Figures 9 and 10 show similar results for the same instance Fict-0660, but using Centre-of-Mass instead of Manual Centroids. 


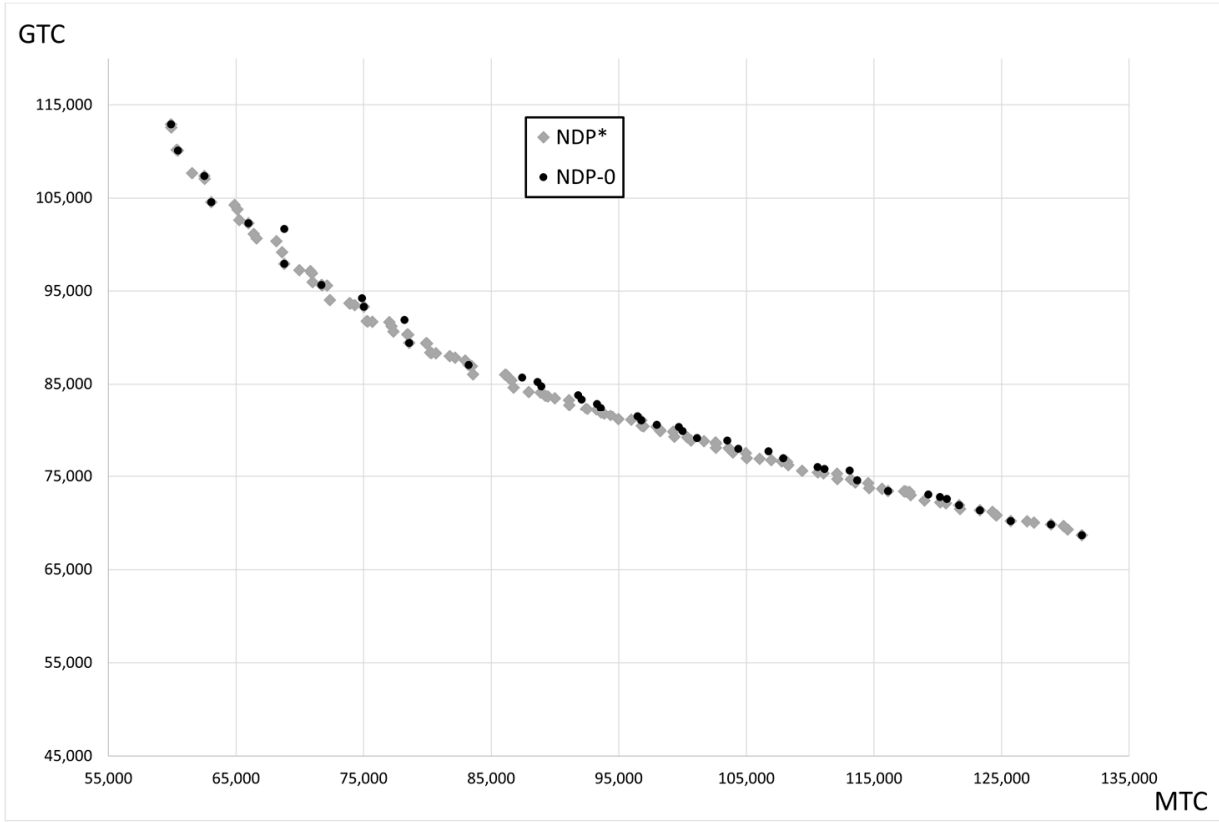

Figure 8. Non-dominated points with Exact and Approximated Formulations with Manual Centroids for instance Fict-0660.

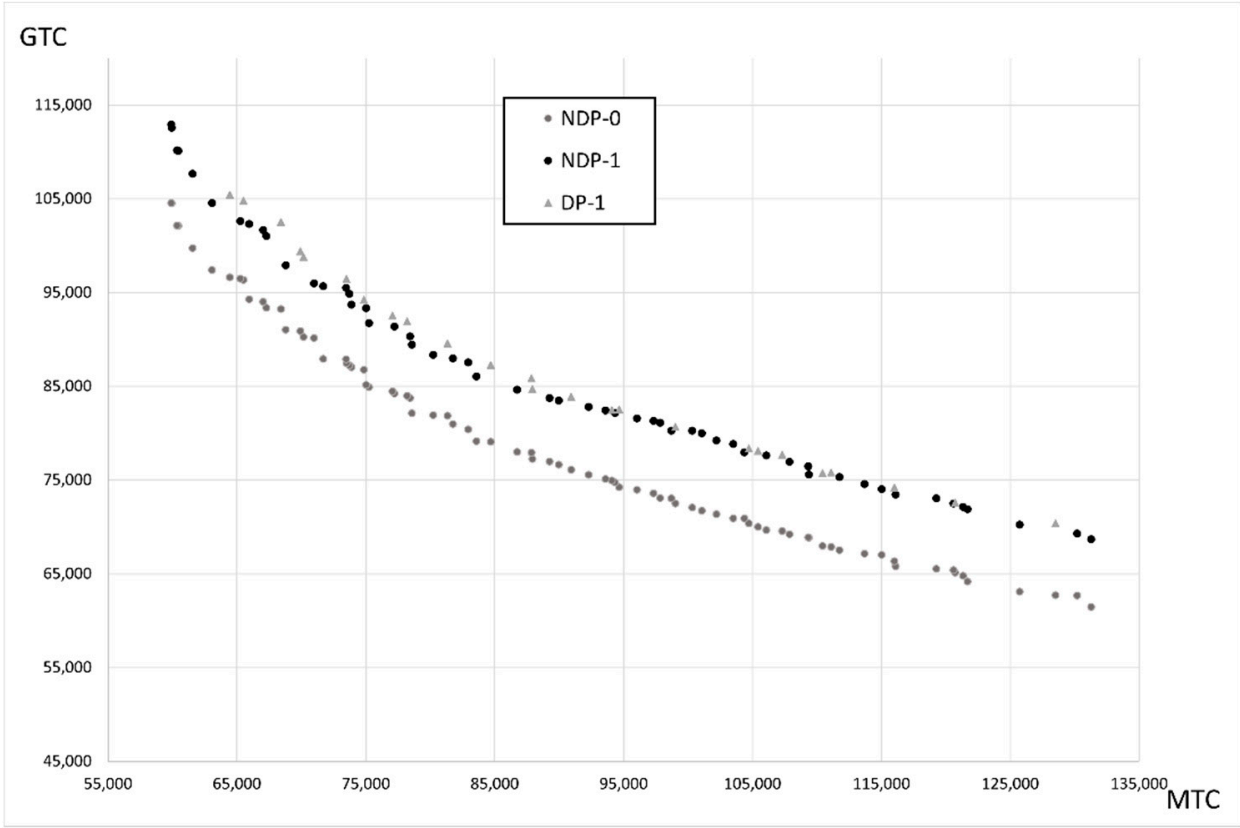

Figure 9. Points associated with efficient solutions for the Aggregated Formulation using Centre-ofMass for instance Fict-0660. 


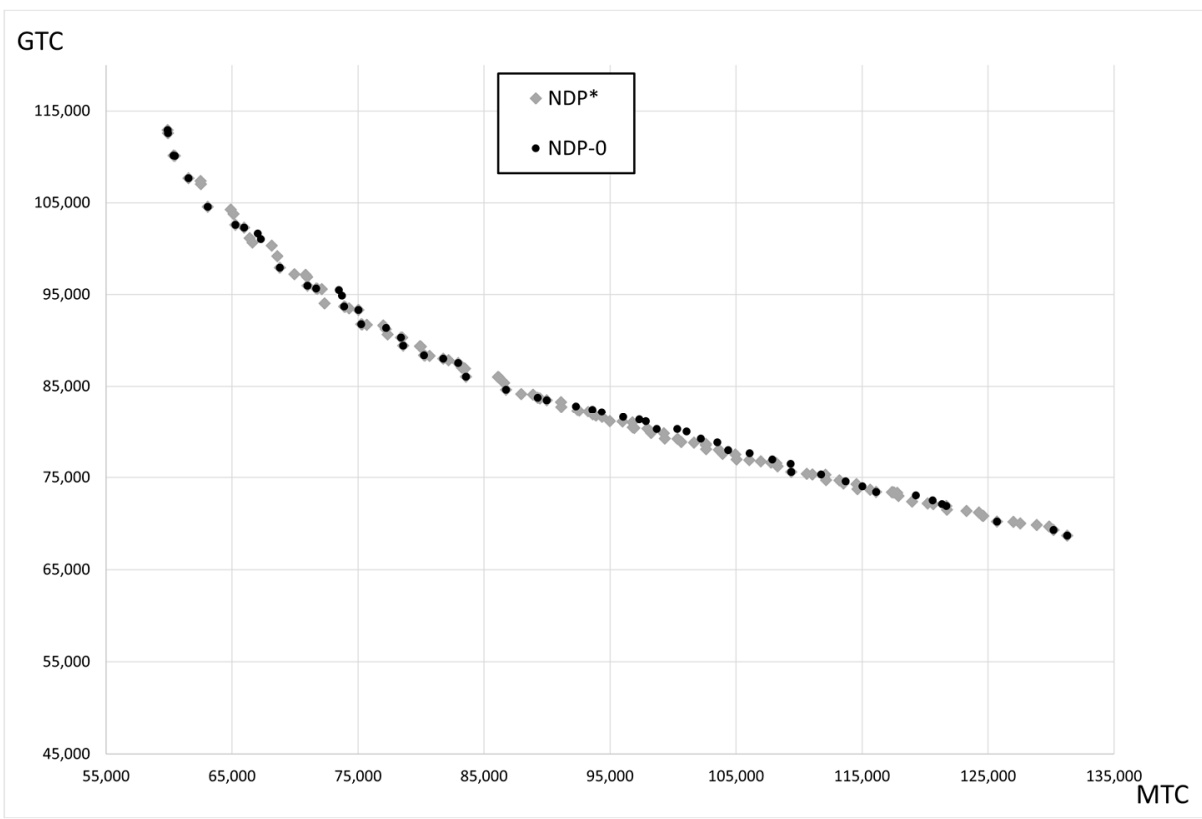

Figure 10. Non-dominated points with Exact and Approximated Formulations with Centre-of-Mass for instance Fict-0660.

Table 2 summarizes all of the previous results for instance Fict-0660, including those results obtained with the Geometric Centroids. In this case, the approximated GTC is an underestimation of the exact GTC, analogously to the Manual Centroid approach. The terminology in Table 2 is as follows:

Average GTC-Error: Average of the relative error of the approximated GTC with respect to the exact GTC for all obtained non-dominated points (exact-approximated). It is positive (negative) when the approximated GTC is lower (higher) than the exact GTC;

$D A 1_{X}$ : Relative DA for a set $X$ with respect to the DA for the Exact Pareto Front;

DA2 $X$ : Relative DA for a set $X$ with respect to the area of the square that defines DA for the ideal point $\left(f_{1}^{\min }, f_{2}^{\min }\right)$, as shown in Figure 5a;

Min. Error with Norm- $\infty$ : Minimum value of the maximum relative errors for each dominated solution;

Min. Error with Norm-2: Minimum value of the Euclidean relative errors for each dominated solution;

Avg1: Average values of all dominated points, including zero values;

Avg2: Average values of all dominated points, excluding zero values.

Notice that the most appropriate outcome when using the Approximated Model is NDP-1 instead of NDP-0, assuming that it is possible to compute the exact GTC for any point of the Approximated Pareto Front, which is not equivalent to solving the Exact Formulation. In concordance with the previous discussion, Table 2 shows that the initial number of non-dominated points obtained with the Approximated Model (NDP-0 $=81,78$, and 80 for the Manual, Geometric, and Centre-of-Mass centroids, respectively) includes points that must be removed from the set of non-dominated points after GTC is exactly recomputed (NDP-0-NDP-1 = 38, 36, and 25 for the Manual, Geometric, and Centre-ofMass centroids, respectively). In other words, when the exact GTC is recomputed for the original set of non-dominated points (NDP-0), there is no guarantee that all points remain as non-dominated points. This result manifests an important inefficiency of using the Approximated Model. Conversely, the Exact Formulation provides the exact set of nondominated points without the need for removing any point (i.e., for the Exact Formulation, $\mathrm{NDP}^{*}=\mathrm{NDP}-1=\mathrm{NDP}-2$ and DP-1 $=\mathrm{DP}-2=0$ ). 
Table 2. Result summary for instance Fict-0660.

\begin{tabular}{|c|c|c|c|c|c|c|c|c|c|c|c|c|c|c|c|c|c|c|}
\hline \multirow[t]{2}{*}{ Formulation } & \multirow[t]{2}{*}{$\begin{array}{l}\text { Centroid } \\
\text { Method }\end{array}$} & \multirow[t]{2}{*}{$\begin{array}{c}\text { Average } \\
\text { GTC- } \\
\text { Error }\end{array}$} & \multirow[t]{2}{*}{ |NDP-0| } & \multirow[t]{2}{*}{ |NDP-1| } & \multirow[t]{2}{*}{$\begin{array}{l}\text { |NDP-1| } \\
1 \\
\mid \text { |NDP-0| }\end{array}$} & \multirow[t]{2}{*}{$\begin{array}{l}\mid \text { NDPP-1| } \\
\text { INDP* }\end{array}$} & \multirow[t]{2}{*}{${ }^{D A} 1_{N D P}-1$} & \multirow[t]{2}{*}{$\mathrm{DA}^{2}{ }_{\mathrm{NDP}}-1$} & \multirow[t]{2}{*}{ |NDP-2| } & \multirow{2}{*}{$\begin{array}{l}\text { |NDP-2| } \\
\mid \\
\mid \text { |NDP-1| }\end{array}$} & \multirow{2}{*}{$\begin{array}{l}\mid \text { NDP-2| } \\
1 \\
\left|N^{*}\right|\end{array}$} & \multirow[t]{2}{*}{ |DP-2| } & \multirow{2}{*}{$\begin{array}{c}\mid \text { DP-2| } \\
\mid \\
\mid \text { |NDP-1| }\end{array}$} & \multirow{2}{*}{$\begin{array}{l}\text { IDP-2| } \\
1 \\
\left|N^{2} P^{*}\right|\end{array}$} & \multicolumn{2}{|c|}{$\begin{array}{l}\text { Min. Error } \\
\text { With } \\
\text { Norm } \infty\end{array}$} & \multicolumn{2}{|c|}{$\begin{array}{l}\text { Min. Error } \\
\text { With } \\
\text { Norm } 2\end{array}$} \\
\hline & & & & & & & & & & & & & & & Avg1 & Avg2 & Avg1 & Avg2 \\
\hline Approx. & $\begin{array}{c}\text { Manual } \\
\text { Gometric }\end{array}$ & $\begin{array}{l}20.6 \% \\
14.8 \%\end{array}$ & $\begin{array}{l}81 \\
78\end{array}$ & $\begin{array}{l}43 \\
42\end{array}$ & $\begin{array}{l}53.1 \% \\
53.8 \%\end{array}$ & $\begin{array}{l}33.9 \% \\
33.1 \%\end{array}$ & $\begin{array}{l}97.1 \% \\
96.8 \%\end{array}$ & $\begin{array}{l}64.3 \% \\
64.1 \%\end{array}$ & $\begin{array}{l}17 \\
17\end{array}$ & $\begin{array}{l}39.5 \% \\
40.5 \%\end{array}$ & $\begin{array}{l}13.4 \% \\
13.4 \%\end{array}$ & $\begin{array}{l}26 \\
25\end{array}$ & $\begin{array}{l}60.5 \% \\
59.5 \%\end{array}$ & $\begin{array}{l}20.5 \% \\
19.7 \%\end{array}$ & $\begin{array}{l}0.47 \% \\
0.55 \%\end{array}$ & $\begin{array}{l}0.78 \% \\
0.92 \%\end{array}$ & $\begin{array}{l}0.54 \% \\
0.62 \%\end{array}$ & $\begin{array}{l}0.89 \% \\
1.50 \%\end{array}$ \\
\hline Exact & $\mathrm{N} / \mathrm{A}$ & $0.0 \%$ & 127 & 127 & $100.0 \%$ & $100.0 \%$ & $100.0 \%$ & $66.3 \%$ & 127 & $100.0 \%$ & $100.0 \%$ & 0 & $0.0 \%$ & $0.0 \%$ & $0.00 \%$ & $0.00 \%$ & $0.00 \%$ & $0.00 \%$ \\
\hline
\end{tabular}


The results in Table 2 also reveal that the utilization of the Centre-of-Mass approach provides a better set of non-dominated points for instance Fict-0660, when compared to the other two methods (Manual and Geometric). This inference is obtained by observing the indicators in this table (i.e., Average GTC-Error, | NDP-1 | , DA1 $1_{X}, \mathrm{DA} 2_{X},|\mathrm{NDP}-2|$, and the error measurements of the dominated points).

All error measurements shown in Table 2 are relatively small for the three types of centroids, having average values less than or equal to $1.05 \%$. Moreover, by observing errors with norm $\infty$, the dominated points obtained with the Approximated Model (DP-2) that do not belong to NDP* present an average deviation of at most $0.92 \%$ in terms of the MTC or GTC (with respect to the points in $\mathrm{NDP}^{*}$ that dominate it). Although norm 2 is an upper bound to norm $\infty$, both norms seem to depict very similar values. Finally, despite the small error measurements, the number of points provided by the Approximated Model (NDP-1) appears to be significantly smaller compared to the points in the Exact Pareto Front NDP* (approximately 37\%).

Similarly, Figures 11 and 12 present the results for instance Real-0820 with Manual and Centre-of-Mass Centroids, respectively. Figure 11 shows that the approximated GTC with Manual Centroids overestimates the exact GTC, as opposed to the above fictitious instance, whereas Figure 12 illustrates that the Approximated Model with Centre-of-Mass centroids underestimates the exact GTC, similar to instance Fict-0660. In other words, the manual approach for determining the centroids in the Approximated Model may present erratic results, which may be explained by the non-systematic and random nature of the Manual Centroid method.

Finally, Figure 13 shows the Approximated Pareto Front (NDP-1) considering two types of centroids, Centre-of-Mass (black dots) and Manual Centroids (unfilled squares), and the Exact Pareto Front, NDP* (grey triangles). Although the number of non-dominated points obtained with the two centroid methods is significantly small (on average, 36\% of the $\mathrm{NDP}^{*}$ ), these sets of points are quite close to the Exact Pareto Front NDP*, where it is difficult to visually distinguish between them.

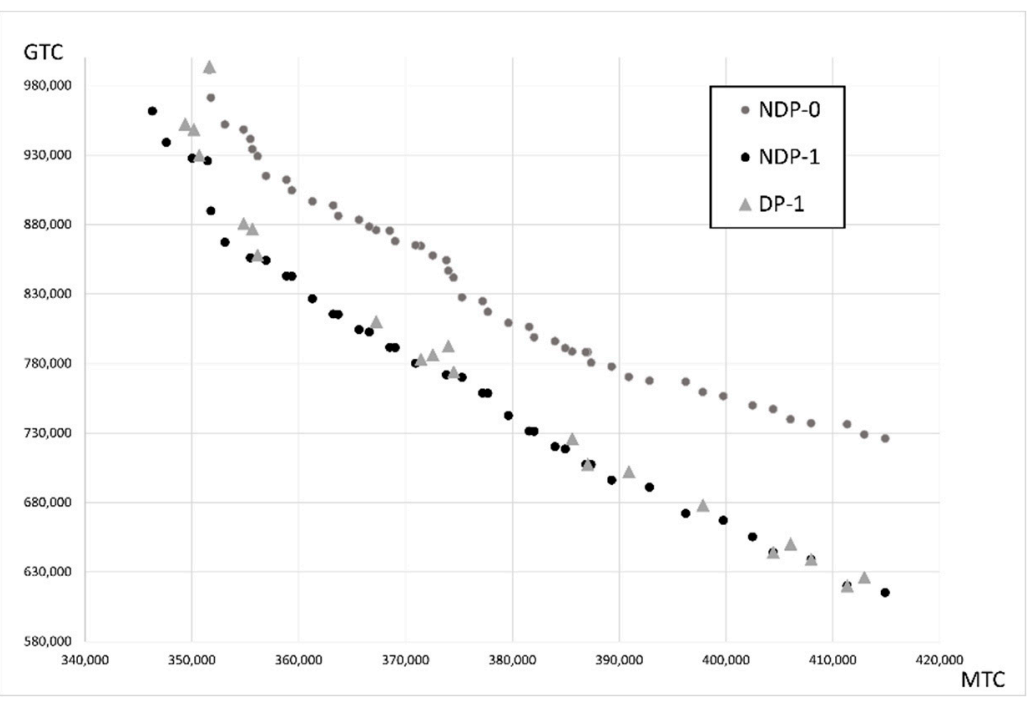

Figure 11. Points associated with efficient solutions for the Aggregated Formulation with Manual Centroids for instance Real-0820. 


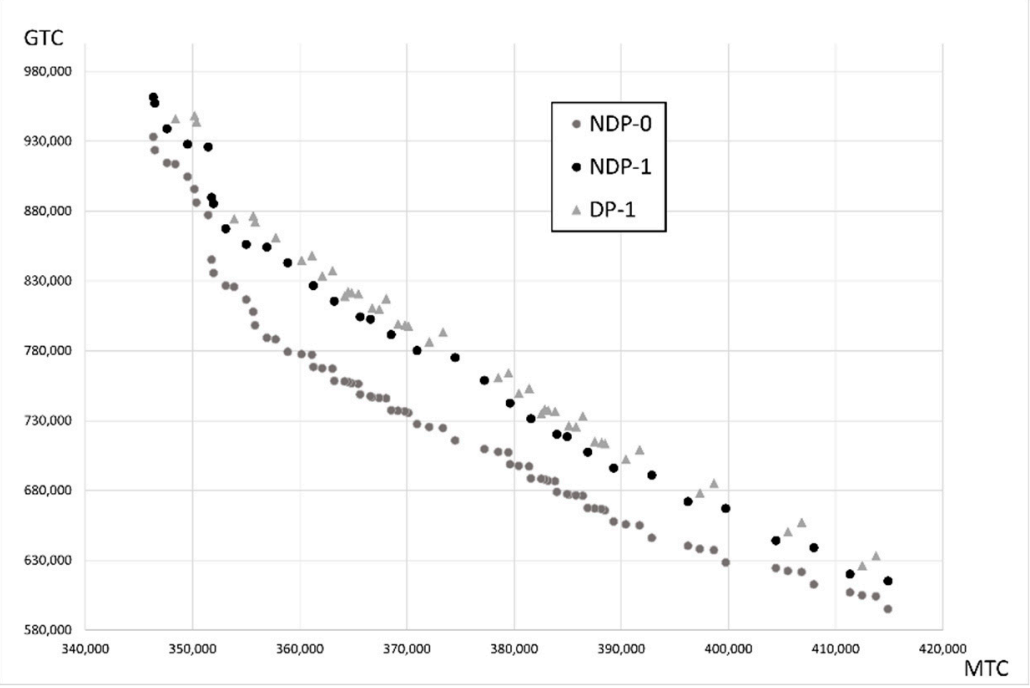

Figure 12. Points associated with efficient solutions for the Aggregated Formulation with Centre-ofMass for instance Real-0820.

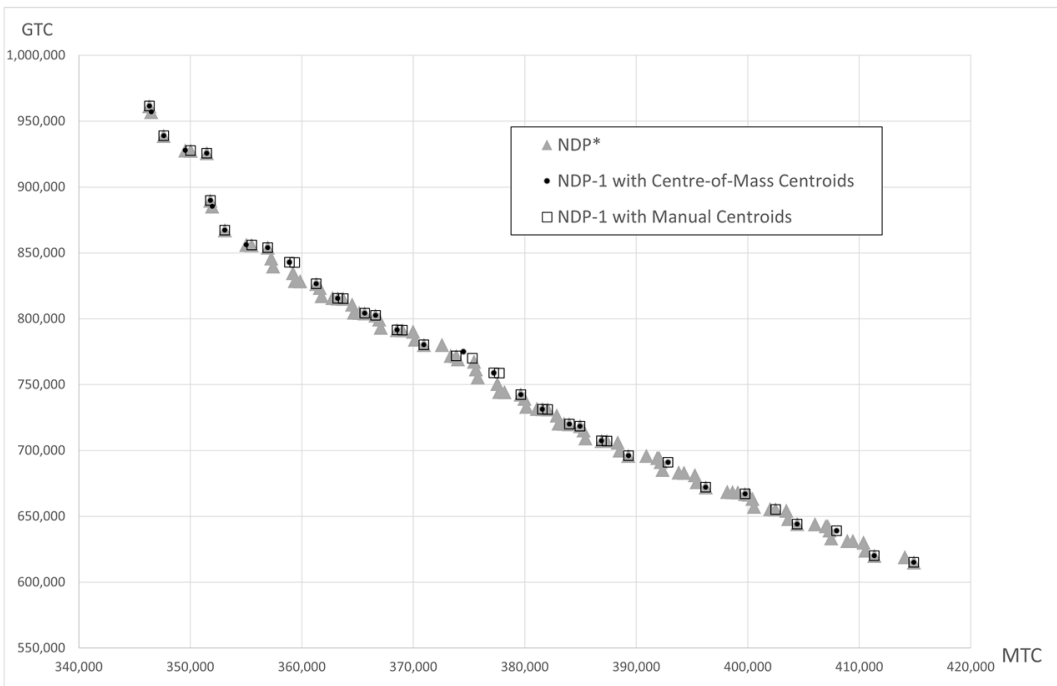

Figure 13. Pareto Fronts for instance Real-0820.

Table 3 presents a summary of the results associated with instance Real-0820, and Tables 4 and 5 summarize the results for instances Fict-0064 and Fict-0004, respectively. These results illustrate an overestimation of the GTC for the Manual Centroids, and an underestimation of the GTC for Centre-of-Mass Centroids for instance Real-0820. In addition, a GTC underestimation is observed for instance Fict-0064 with Manual and Centre-of-Mass Centroids, as shown in Table 4 and Figure 14. This underestimation is not clear for the case of the Geometric Centroids. 
Table 3. Result summary for instance Real-0820.

\begin{tabular}{|c|c|c|c|c|c|c|c|c|c|c|c|c|c|c|c|c|c|c|c|}
\hline \multirow[t]{2}{*}{ Formulation } & \multirow[t]{2}{*}{$\begin{array}{l}\text { Centroid } \\
\text { Method }\end{array}$} & \multirow{2}{*}{$\begin{array}{c}\text { Average } \\
\text { GTC- } \\
\text { Error }\end{array}$} & \multirow{2}{*}{$\begin{array}{l}\text { Avg. Abs. } \\
\text { GTC- } \\
\text { Error }\end{array}$} & \multirow[t]{2}{*}{ |NDP-0| } & \multirow[t]{2}{*}{ |NDP-1| } & \multirow{2}{*}{$\begin{array}{l}\mid \text { NDP-1| } \\
1 \\
\mid \text { INDP-0| }\end{array}$} & \multirow{2}{*}{$\begin{array}{c}\text { INDP-1| } \\
\quad \\
\left|N^{*} P^{*}\right|\end{array}$} & \multirow[t]{2}{*}{ DA1 $1_{\text {NDP-1 }}$} & \multirow[t]{2}{*}{${ }^{D A}{ }^{2}$ NDP-1 } & \multirow[t]{2}{*}{ |NDP-2| } & \multirow{2}{*}{$\begin{array}{l}|\mathrm{NDP}-2| \\
1 \\
|\mathrm{NDP}-1|\end{array}$} & \multirow{2}{*}{$\begin{array}{c}\mid \text { NDP-2| } \\
I \\
\left|N^{*} P^{*}\right|\end{array}$} & \multirow[t]{2}{*}{$|\mathrm{DP}-2|$} & \multirow{2}{*}{$\begin{array}{c}\text { |DP-2| } \\
1 \\
\mid \text { |NDP-1| }\end{array}$} & \multirow{2}{*}{$\begin{array}{c}|\mathrm{DP}-2| \\
1 \\
\mid \\
\left|\mathrm{NDP}^{*}\right|\end{array}$} & \multicolumn{2}{|c|}{$\begin{array}{l}\text { Min. Error } \\
\text { with } \\
\text { Norm } \infty\end{array}$} & \multicolumn{2}{|c|}{$\begin{array}{l}\text { Min. Error } \\
\text { with } \\
\text { Norm 2 }\end{array}$} \\
\hline & & & & & & & & & & & & & & & & Avg1 & Avg2 & Avg1 & Avg2 \\
\hline \multirow{2}{*}{ Approx. } & Manual & $-10.76 \%$ & $10.76 \%$ & 59 & 38 & $64.4 \%$ & $39.2 \%$ & $97.7 \%$ & $59.9 \%$ & 31 & $81.58 \%$ & $31.96 \%$ & 7 & $18.4 \%$ & $7.2 \%$ & $0.10 \%$ & $0.57 \%$ & $0.12 \%$ & $0.65 \%$ \\
\hline & $\begin{array}{l}\text { Centre of } \\
\text { Mass }\end{array}$ & $6.32 \%$ & $6.32 \%$ & 77 & 32 & $41.6 \%$ & $33.0 \%$ & $97.6 \%$ & $59.8 \%$ & 27 & $84.38 \%$ & $27.84 \%$ & 5 & $15.6 \%$ & $5.2 \%$ & $0.09 \%$ & $0.60 \%$ & $0.11 \%$ & $0.67 \%$ \\
\hline Exact & $\mathrm{N} / \mathrm{A}$ & $0.00 \%$ & $0.00 \%$ & 97 & 97 & $100.0 \%$ & $100.0 \%$ & $100.0 \%$ & $61.2 \%$ & 97 & $100.00 \%$ & $100.00 \%$ & 0 & $0.0 \%$ & $0.0 \%$ & $0.00 \%$ & $0.00 \%$ & $0.00 \%$ & $0.00 \%$ \\
\hline
\end{tabular}

Table 4. Result summary for instance Fict-0064.

\begin{tabular}{|c|c|c|c|c|c|c|c|c|c|c|c|c|c|c|c|c|c|c|c|}
\hline \multirow[t]{2}{*}{ Formulation } & \multirow{2}{*}{$\begin{array}{l}\text { Centroid } \\
\text { Method }\end{array}$} & \multirow{2}{*}{$\begin{array}{c}\text { Average } \\
\text { GTC- } \\
\text { Error }\end{array}$} & \multirow{2}{*}{$\begin{array}{l}\text { Avg. Abs. } \\
\text { GTC- } \\
\text { Error }\end{array}$} & \multirow[t]{2}{*}{ |NDP-0| } & \multirow[t]{2}{*}{ INDP-1| } & \multirow{2}{*}{$\begin{array}{c}\text { |NDP-1| } \\
\mid \\
\mid \text { |NDP-0| }\end{array}$} & \multirow{2}{*}{$\begin{array}{c}\mid \text { NDP-1| } \\
I \\
\left|N^{*} P^{*}\right|\end{array}$} & \multirow[t]{2}{*}{${ }^{D A 1}{ }_{N D P}-1$} & \multirow[t]{2}{*}{${ }^{\mathrm{DA}}{ }_{\mathrm{NDP}}-1$} & \multirow[t]{2}{*}{$\mid$ NDP-2| } & \multirow{2}{*}{$\begin{array}{c}\text { |NDP-2| } \\
\mid \\
\mid \text { |NDP-1| }\end{array}$} & \multirow{2}{*}{$\begin{array}{l}\mid \text { INDP-2| } \\
\mid \\
\left|N^{*} P^{*}\right|\end{array}$} & \multirow[t]{2}{*}{$\mid$ DP-2| } & \multirow{2}{*}{$\begin{array}{c}\mid \text { DP-2| } \\
\quad \\
\mid \text { |NDP-1| }\end{array}$} & \multirow{2}{*}{$\begin{array}{c}\text { IDP-2| } \\
\text { I } \\
\text { |NDP*1 }\end{array}$} & \multicolumn{2}{|c|}{$\begin{array}{l}\text { Min. Error } \\
\text { with } \\
\text { Norm } \infty\end{array}$} & \multicolumn{2}{|c|}{$\begin{array}{c}\text { Min. Error } \\
\text { with } \\
\text { Norm } 2\end{array}$} \\
\hline & & & & & & & & & & & & & & & & Avg1 & Avg2 & Avg1 & Avg2 \\
\hline \multirow[b]{2}{*}{ Approx. } & $\begin{array}{c}\text { Manual } \\
\text { Gemertic }\end{array}$ & $\begin{array}{r}5.19 \% \\
-050 \% \%\end{array}$ & $5.19 \%$ & $\begin{array}{l}99 \\
103\end{array}$ & $\begin{array}{l}57 \\
59\end{array}$ & $\begin{array}{l}57.6 \% \\
573 \%\end{array}$ & $\begin{array}{l}35.4 \% \\
336 \% \%\end{array}$ & $\begin{array}{l}98.5 \% \\
98.6 \%\end{array}$ & $\begin{array}{l}55.5 \% \\
55.5 \%\end{array}$ & 31 & $\begin{array}{l}54.4 \% \\
552 \%\end{array}$ & $\begin{array}{l}19.3 \% \\
109 \%\end{array}$ & 26 & $\begin{array}{l}45.6 \% \% \\
458 \% \%\end{array}$ & $\begin{array}{l}16.1 \% \\
168 \% \%\end{array}$ & $\begin{array}{l}0.30 \% \\
0.28 \%\end{array}$ & $0.63 \%$ & $\begin{array}{l}0.34 \% \\
0.3 \% \%\end{array}$ & $0.73 \%$ \\
\hline & $\begin{array}{l}\text { Geometric } \\
\text { Centre }\end{array}$ & $-0.50 \%$ & $2.92 \%$ & 103 & 59 & $57.3 \%$ & $36.6 \%$ & $98.6 \%$ & $55.5 \%$ & 32 & $54.2 \%$ & $19.9 \%$ & 27 & $45.8 \%$ & $16.8 \%$ & $0.28 \%$ & $0.61 \%$ & $0.33 \%$ & $0.71 \%$ \\
\hline Exact & $\mathrm{N} / \mathrm{A}$ & $0.00 \%$ & $0.00 \%$ & 161 & 161 & $100.0 \%$ & $100.0 \%$ & $100.0 \%$ & $56.3 \%$ & 161 & $100.0 \%$ & $100.0 \%$ & 0 & $0.0 \%$ & $0.0 \%$ & $0.00 \%$ & $0.00 \%$ & $0.00 \%$ & $0.00 \%$ \\
\hline
\end{tabular}

Table 5. Result summary for instance Fict-0004.

\begin{tabular}{|c|c|c|c|c|c|c|c|c|c|c|c|c|c|c|c|c|c|c|c|}
\hline \multirow[t]{2}{*}{ Formulation } & \multirow[t]{2}{*}{$\begin{array}{l}\text { Centroid } \\
\text { Method }\end{array}$} & \multirow{2}{*}{$\begin{array}{c}\text { Average } \\
\text { GTC- } \\
\text { Error }\end{array}$} & \multirow{2}{*}{$\begin{array}{l}\text { Avg. Abs. } \\
\text { GTC- } \\
\text { Error }\end{array}$} & \multirow[t]{2}{*}{ |NDP-0| } & \multirow[t]{2}{*}{ |NDP-1| } & \multirow{2}{*}{$\begin{array}{l}\mid \text { NDP-1| } \\
\mid \\
\mid \text { |NDP-0| }\end{array}$} & \multirow{2}{*}{$\begin{array}{l}\text { INDP-1| } \\
\mid-1 \\
\left|N^{*} P^{*}\right|\end{array}$} & \multirow[t]{2}{*}{${ }^{D A} 1_{N D P-1}$} & \multirow[t]{2}{*}{ DA2 ${ }^{\text {NDP-1 }}$} & \multirow[t]{2}{*}{ |NDP-2| } & \multirow{2}{*}{$\begin{array}{l}|\mathrm{NDP}-2| \\
1 \\
|\mathrm{NDP}-1|\end{array}$} & \multirow{2}{*}{$\begin{array}{l}\text { |NDP-2| } \\
1 \\
\left|\mathrm{NDP}^{*}\right|\end{array}$} & \multirow[t]{2}{*}{ |DP-2| } & \multirow{2}{*}{$\begin{array}{c}\mid \text { DP-2| } \\
\mid \\
\mid \text { INDP-1| }\end{array}$} & \multirow{2}{*}{$\begin{array}{l}|D P-2| \\
\mid \\
\left|N^{*} P^{*}\right|\end{array}$} & \multicolumn{2}{|c|}{$\begin{array}{l}\text { Min. Error } \\
\text { with } \\
\text { Norm } \infty\end{array}$} & \multicolumn{2}{|c|}{$\begin{array}{l}\text { Min. Error } \\
\text { with } \\
\text { Norm 2 }\end{array}$} \\
\hline & & & & & & & & & & & & & & & & Avg1 & Avg2 & Avg1 & Avg2 \\
\hline Approx. & $\begin{array}{l}\text { Manual } \\
\text { Geometric }\end{array}$ & $\begin{array}{l}0.28 \% \\
-4.86 \%\end{array}$ & $\begin{array}{l}3.51 \% \\
6.86 \%\end{array}$ & $\begin{array}{l}41 \\
40\end{array}$ & $\begin{array}{l}30 \\
30\end{array}$ & $\begin{array}{l}73.2 \% \\
75.0 \%\end{array}$ & $\begin{array}{l}40.0 \% \\
40.0 \%\end{array}$ & $\begin{array}{l}97.4 \% \\
97.4 \%\end{array}$ & $\begin{array}{l}64.7 \% \\
64.7 \%\end{array}$ & $\begin{array}{l}22 \\
22\end{array}$ & $\begin{array}{l}73.3 \% \\
73.3 \%\end{array}$ & $\begin{array}{l}29.3 \% \\
29.3 \%\end{array}$ & $\begin{array}{l}8 \\
8\end{array}$ & $\begin{array}{l}26.7 \% \\
26.7 \%\end{array}$ & $\begin{array}{l}26.7 \% \\
26.7 \%\end{array}$ & $\begin{array}{l}0.52 \% \\
0.52 \%\end{array}$ & $\begin{array}{l}1.94 \% \\
1.94 \%\end{array}$ & $\begin{array}{l}0.59 \% \\
0.59 \%\end{array}$ & $\begin{array}{l}2.19 \% \\
2.19 \%\end{array}$ \\
\hline Exact & $\mathrm{N} / \mathrm{A}$ & $0.00 \%$ & $0.00 \%$ & 75 & 75 & $100.0 \%$ & $100.0 \%$ & $100.0 \%$ & $66.4 \%$ & 75 & $100.0 \%$ & $100.0 \%$ & 0 & $0.0 \%$ & $0.0 \%$ & $0.00 \%$ & $0.00 \%$ & $0.00 \%$ & $0.00 \%$ \\
\hline
\end{tabular}




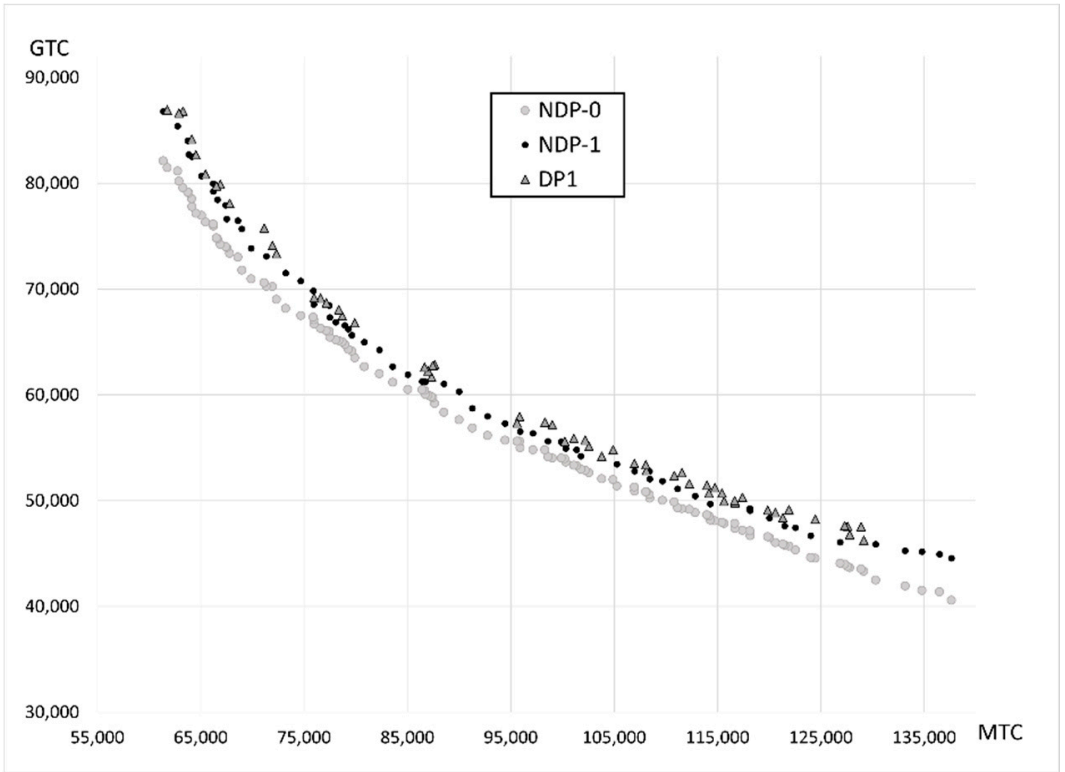

Figure 14. Pareto Fronts for the Aggregated Formulation with Centre-of-Mass Centroids for instance Fict-0064.

In Tables 3-5, column "Avg. Abs. GTC-Error" is added to show the average of the absolute GTC errors. This indicator becomes relevant in some cases, where some points obtained with the Approximated Model (NDP-0) present a GTC overestimation, and other points of the same set present a GTC underestimation. Thus, columns "Average GTC-Error" and "Avg. Abs. GTC-Error" may yield different absolute values, such as for the Geometric Centroids for instance Fict-0064 (See Table 4), and for all types of centroids for instance Fict-0004 (see Table 5 and Figure 15).

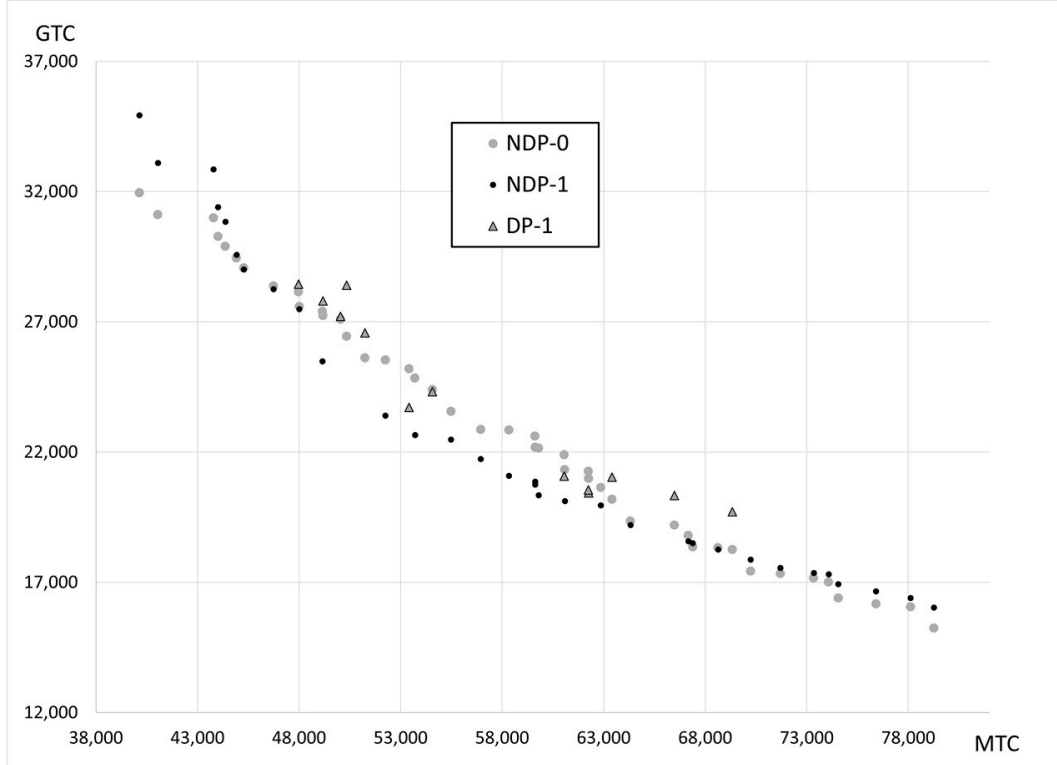

Figure 15. Pareto Fronts for the Aggregated Formulation with Centre-of-Mass Centroids for instance Fict-0004. 
Two main types of errors related to the GTC estimation for the Approximated Model may be distinguished. One type of error, Error Type A, consists of an underestimation of the GTC between the Centre-of-Mass centroids and the ports compared to the actual GTC between the real demand locations and the ports for the Exact Formulation, as shown in Figure 16. This type of error only applies for the Centre-of-Mass Centroids, since the other two methods may present an erratic error behavior. In other words, these methods may yield either an underestimation or overestimation of the GTC, whose behavior cannot be anticipated nor systematically studied.

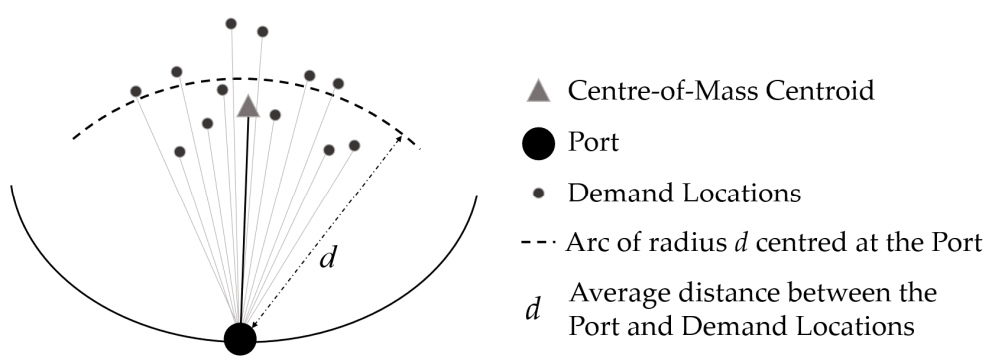

Figure 16. Error Type A.

The other type of error, Error Type B, relates to the centroid demand splitting, when its associated port is not selected. In this situation, the Approximated Model assumes that this demand is homogeneously split among the selected ports (for all types of centroids), as indicated in Figure 17. This assumption does not apply when either one or all ports are selected. If only one port is selected, then the whole island demand is collected through this single port. Whereas, if all of the ports are selected, then the demand of each centroid is fully collected through its associated port, and thus, this type of error is non-existent.

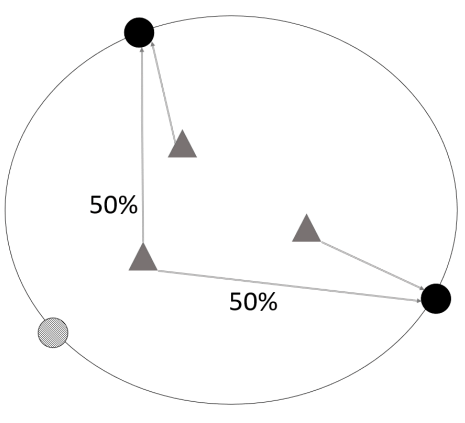

(a)

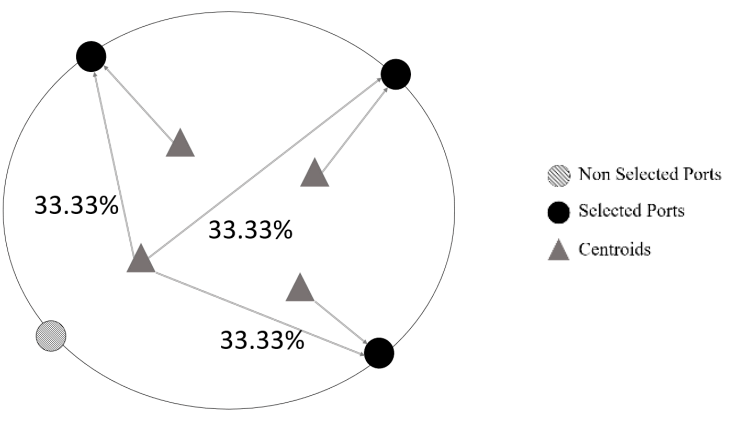

(b)

Figure 17. Error Type B associated with demand splitting, for islands with three and four ports: (a) demand splitting for a three-port island; (b) demand splitting for a four-port island.

The aforementioned approximated manner of splitting the demand of a centroid may be acceptable in the case that two out of three available ports are selected, as in Figure 17a. On the contrary, as Figure 17b suggests, this assumption does not seem to be reasonable for a four-port island, since the centroid demand should be more concentrated towards the two nearest selected ports (as opposed to the homogenous splitting assumption). Consequently, this type of error produces a GTC overestimation.

According to the previous discussion, instances containing islands with fewer than four ports mainly present Error Type A, which yields a significant GTC underestimation (only for the Centre-of-Mass Centroid method). This underestimation is clearly observed in Figures 14 and 17 and Tables 1 and 2. Conversely, the two types of errors may be significant for instances with four or more ports, producing a combination of GTC in Figures 14 and 15 and Tables 4 and 5 . 


\subsection{Computational Performance Evaluation with Larger Instances}

This section presents a discussion on the results obtained for the set of 20 instances (10 fictitious and 10 real). The fictitious instances contain seven two-port islands, seven three-port islands, and three four-port islands (instances Fict-0773-01 through Fict-0773-10), and the real instances contain eight islands of one port, eight islands of two ports, and two islands of three ports (instances Real-8820-01 through Real-8820-10).

Table 6 presents a summary of the results when solving the 20 instances with the Approximated Model and the Exact Formulation, assuming the same notation of the previous tables. In this case, the Approximated Model is solved considering only Centre-ofMass Centroids. Appendix A graphically shows the set of dominated points obtained with the two formulations for instances Real-8820-01 through Real-8820-10 with the Manual Centroids method. Table 6 shows that the number of non-dominated points using the Exact Formulation (NDP*) is significantly larger for the fictitious instances than for the real instances, since the former has considerably more nodes (51 vs. 30). Therefore, a larger number of nodes produces significantly more points in the objective space due to the combinatorial nature of the problem.

In addition, the average GTC error is considerably larger for the fictitious instances (7.63\% vs. $3.71 \%$ ), which could be mainly explained by a smaller Error Type A in the real instances. Notice that the real instances may not present a significant Error Type B because these instances do not have four-port islands (as discussed in Section 4.1). These smaller GTC errors for the real instances yield better results in terms of the quality of the obtained sets of non-dominated points (NDP-1), which is observed in the performance indicators ( $|\mathrm{NDP}-1|$ and $|\mathrm{NDP}-2|)$. The $\mathrm{DA}_{\mathrm{NDP}-1}$ is the only indicator that performs similarly for both sets of fictitious and real instances. For example, on average, 37.64\% of the points obtained for the fictitious instances remain as non-dominated after GTC is recomputed ( $\mid$ NDP-1 | / | NDP-0 | ), while these points also represent $33.83 \%$ of the Exact Pareto Front ( $\mid$ NDP-1 | / | NDP*|). These percentages rise to $50.51 \%$ and $57.05 \%$ for the real instances, respectively. Furthermore, the algorithm obtains, on average, $9.9 \%$ of the points in the Exact Pareto Front for the fictitious instances, and $28.7 \%$ for the real instances ( | NDP-2 | / |NDP*|).

Overall, the performance indicators are quite similar within each instance group. For example, the values of the "Avg. GTC Error" present a coefficient of variation close to 0.1 for the real instances and 0.07 for the fictitious instances, denoting the low dispersion of these values. Additionally, | NDP-1 I / NDP*| presents a coefficient of variation close to 0.09 and 0.1 for the real and fictitious instances, respectively.

Concurring with the results associated with the smaller instances in Section 4.1, all error measurements in Table 6 show that all dominated points (DP-2) obtained with the Approximated Model are relatively close to the exact set of non-dominated points (on average at most $1 \%$ in GTC or MTC, according to norm $\infty$ ).

For the same groups of real and fictitious instances, Table 7 adds the following notation:

$\mathrm{N}$ : Number of executions within the AUGMECON2 algorithm (i.e., a mono-objective optimization);

Time: Total computing time to complete the $N$ executions;

$\Delta 1$ : Relative variation in the indicator Time/N (Exact vs. Approximated), which relates to the time variation when solving a mono-objective optimization problem;

$\Delta 2$ : Relative variation in the indicator Time/NDP-0 (Exact vs. Approximated), which relates to the time variation for obtaining non-dominated points prior to the GTC recomputation;

13: Relative variation in the indicator Time/NDP-1 (Exact vs. Approximated), which relates to the time variation for obtaining non-dominated points after the GTC re-computation.

Notice that $\mathrm{NDP}^{*}=\mathrm{NDP}-0=\mathrm{NDP}-1$ for the Exact Formulation, since the nondominated points obtained with the Exact Formulation actually belong to the Exact Pareto Front. 
Table 6. Summary of the performance indicators.

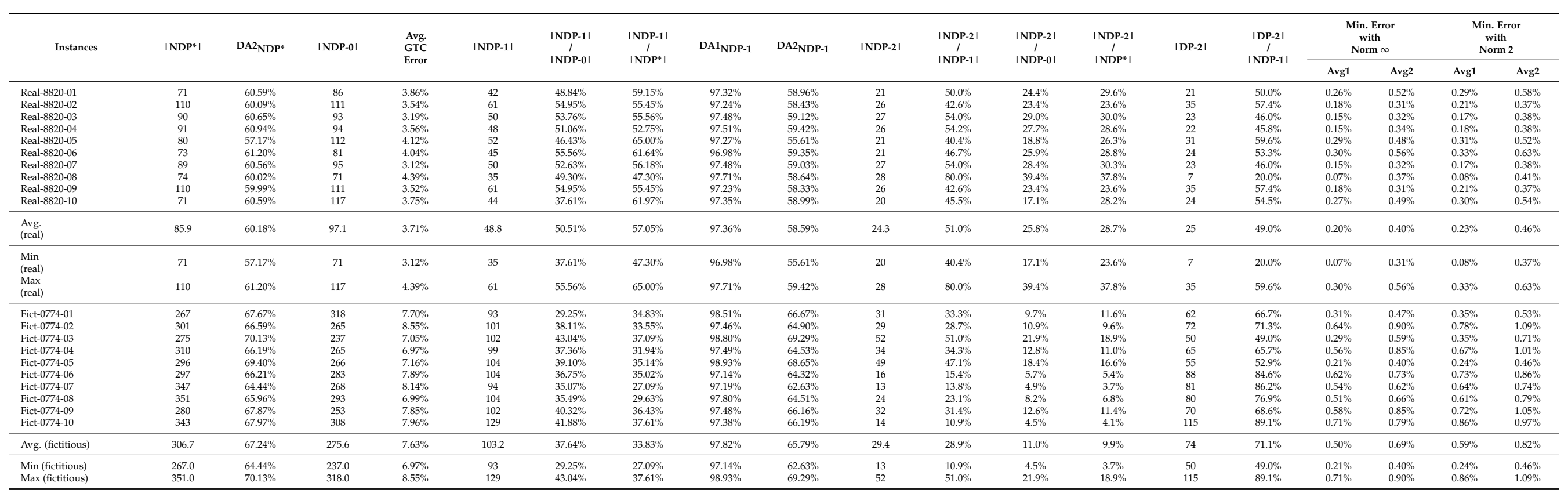


The efficiency of employing the Approximated Model to obtain non-dominated points performs slightly better for the real instances. $35.8 \%$ of the executions provide non-dominated points (NDP-1) for the fictitious instances, whereas $42 \%$ of the executions provide non-dominated points for the real instances ( $|\mathrm{NDP}-1| / N)$. These results may be explained by a better GTC estimation for the real instances than for the fictitious instances, which is observed in Table 6 through the column "Avg. GTC-Error".

The average computing times are significantly larger for the fictitious instances than for the real instances (17,742 s vs. $673 \mathrm{~s}$ for the Approximated Model, and 22,712 s vs. $984 \mathrm{~s}$ for the Exact Formulation). Obviously, this is explained by the instance sizes (51 ports for the fictitious instances vs. 30 ports for the real instances). Additionally, as may be expected, the Exact Formulation is more time-consuming than the Approximated Model (984 s vs. $673 \mathrm{~s}$ for the real instances, and 22,712 s vs. 17,742 s for the fictitious instances). However, the advantage of the Approximated Model with respect to the Exact Formulation in terms of the computing time decreases for the fictitious instances. On average, the Approximated Model consumes 32\% less time than the Exact Formulation for the real instances (673 s vs. $984 \mathrm{~s}$ ), whereas, for the fictitious instances, the Approximated Model consumes $22 \%$ less time than the Exact Formulation (17,742 s vs. 22,712 s). These results are due to the fact that the complexity of the Approximated Model is significantly conditioned by the number of ports per island (in addition to the total number of ports), which, in this case, is larger for the fictitious instances (two, three, and four ports per island for the fictitious islands vs. one, two, and three ports per island for the real instances).

The previous statement holds since Expression (1.2), which is employed to approximate the GTC, relies on a combinatory of existing ports that determine all possible groups of selected ports for each island. Conversely, the complexity of the Exact Formulation is less sensitive to the number of ports per island, since it depends on the combination of ports and demand locations through a linear formulation in Expression (2.2).

The most relevant result observed in Table 7 is the significant improvement regarding the time required for providing a non-dominated point $\left(\mathrm{NDP}^{*}\right)$ for the Exact Formulation and NDP-1 for the Approximated Model ( $\Delta 3$ ). As discussed in Section 4, NDP-1 is obtained after the GTC is recomputed and the resulting dominated points are removed from the set. For the real instances, this time reduction is, on average, $13.7 \%$, whereas, for the fictitious instances, the time reduction is, on average, $61.1 \%$. This result is strictly related to a better algorithm performance when the Exact Formulation is employed, where only in $41.96 \%$ of the executions $(N)$ with the Approximated Model provide a non-dominated point (i.e., NDP-1) for the real instances and $35.79 \%$ for the fictitious instances. In contrast, when employing the Exact Formulation, the AUGMECON2 algorithm provides, on average, a non-dominated point (NDP-1 $=\mathrm{NDP}^{*}$ ) in $82.62 \%$ of the executions for the real instances, and in $94.94 \%$ of the executions for the fictitious instances. As a conclusion, although the quality of the set of non-dominated points obtained when employing the Approximated Model may be considered as acceptable, when observing the results in Table 5, the number of non-dominated points obtained with similar computing times is significantly lower for the Approximated Model than for the proposed novel Exact Formulation (i.e., on average, the size of NDP- 1 is around $45 \%$ of the size of NDP*, of which, only $40 \%$ finally belongs to $\mathrm{NDP}^{*}$. 
Table 7. Computing performance of Approximated Model with Centre-of-Mass method.

\begin{tabular}{|c|c|c|c|c|c|c|c|c|c|c|c|c|c|c|c|c|c|c|}
\hline \multirow[b]{2}{*}{ Instance } & \multicolumn{7}{|c|}{ Approximated Model with Centre-of-Mass Centroids } & \multicolumn{5}{|c|}{ Exact Formulation } & \multirow[b]{2}{*}{$\Delta \mathbf{1}$} & \multirow[b]{2}{*}{$\Delta 2$} & \multirow[b]{2}{*}{$\Delta 3$} & \multicolumn{2}{|c|}{$\begin{array}{c}\text { Approximated } \\
\text { Model }\end{array}$} & \multirow{2}{*}{$\begin{array}{c}\begin{array}{c}\text { Exact } \\
\text { Formulation }\end{array} \\
\text { | NDP-0 |/N } \\
= \\
\text { | NDP-1 |/N } \\
\end{array}$} \\
\hline & $\mathbf{N}$ & $\begin{array}{l}\text { Time } \\
\text { (sec) }\end{array}$ & $\begin{array}{c}\text { Time } \\
/ \\
\mathbf{N} \\
\end{array}$ & |NDP-0 | & $\begin{array}{c}\text { Time } \\
\text { I } \\
\text { |NDP-0 | }\end{array}$ & | NDP-1 | & $\begin{array}{c}\text { Time } \\
\text { I } \\
\text { | NDP-1 I }\end{array}$ & $\mathbf{N}$ & $\begin{array}{l}\text { Time } \\
\text { (s) }\end{array}$ & $\begin{array}{c}\text { Time } \\
1 \\
\mathbf{N} \\
\end{array}$ & $\begin{array}{c}\text { | NDP-0 | } \\
= \\
\mid \begin{array}{c}= \\
\text { |NDP-1 | }\end{array}\end{array}$ & $\begin{array}{c}\text { Time } \\
\text { I } \\
\text { |NDP-1 I } \\
\end{array}$ & & & & $\begin{array}{c}\mid \text { NDP-0 | } \\
I \\
\mathbf{N} \\
\end{array}$ & $\begin{array}{c}\mid \text { NDP-1 | } \\
\text { / } \\
\mathbf{N} \\
\end{array}$ & \\
\hline Real-8820-02 & 133 & 830 & 6.2 & 111 & 7.5 & 61 & 13.6 & 138 & 1275 & 9.2 & 110 & 11.6 & $48.0 \%$ & $54.9 \%$ & $-14.9 \%$ & $83.46 \%$ & $45.86 \%$ & $79.71 \%$ \\
\hline Real-8820-03 & 124 & 728 & 5.9 & 93 & 7.8 & 50 & 14.6 & 104 & 1040 & 10.0 & 90 & 11.6 & $70.4 \%$ & $47.7 \%$ & $-20.6 \%$ & $75.00 \%$ & $40.32 \%$ & $86.54 \%$ \\
\hline Real-8820-04 & 123 & 626 & 5.1 & 94 & 6.7 & 48 & 13.0 & 107 & 895 & 8.4 & 91 & 9.8 & $64.3 \%$ & $47.6 \%$ & $-24.6 \%$ & $76.42 \%$ & $39.02 \%$ & $85.05 \%$ \\
\hline Real-8820-05 & 140 & 1051 & 7.5 & 112 & 9.4 & 52 & 20.2 & 104 & 1150 & 11.1 & 80 & 14.4 & $47.3 \%$ & $53.2 \%$ & $-28.9 \%$ & $80.00 \%$ & $37.14 \%$ & $76.92 \%$ \\
\hline Real-8820-06 & 98 & 464 & 4.7 & 81 & 5.7 & 45 & 10.3 & 88 & 851 & 9.7 & 73 & 11.7 & $104.3 \%$ & $103.5 \%$ & $13.1 \%$ & $82.65 \%$ & $45.92 \%$ & $82.95 \%$ \\
\hline Real-8820-07 & 112 & 507 & 4.5 & 95 & 5.3 & 50 & 10.1 & 108 & 905 & 8.4 & 89 & 10.2 & $84.9 \%$ & $90.3 \%$ & $0.2 \%$ & $84.82 \%$ & $44.64 \%$ & $82.41 \%$ \\
\hline Real-8820-09 & 121 & 699 & 5.8 & 111 & 6.3 & 61 & 11.5 & 132 & 1167 & 8.8 & 110 & 10.6 & $53.0 \%$ & $68.5 \%$ & $-7.4 \%$ & $91.74 \%$ & $50.41 \%$ & $83.33 \%$ \\
\hline Real-8820-10 & 138 & 842 & 6.1 & 117 & 7.2 & 44 & 19.1 & 86 & 817 & 9.5 & 71 & 11.5 & $55.7 \%$ & $59.9 \%$ & $-39.9 \%$ & $84.78 \%$ & $31.88 \%$ & $82.56 \%$ \\
\hline Avg. & 117.2 & 673 & 5.7 & 97.1 & 6.8 & 49 & 13.81 & 104.1 & 984 & 9.5 & 85.9 & 11.53 & $69.6 \%$ & $70.6 \%$ & $-13.7 \%$ & $83.08 \%$ & $41.96 \%$ & $82.62 \%$ \\
\hline Min & 81.0 & 464 & 4.5 & 71.0 & 5.3 & 35 & 10.1 & 84 & 731 & 8.4 & 71 & 9.8 & $47.3 \%$ & $47.6 \%$ & $-39.9 \%$ & $75.00 \%$ & $31.88 \%$ & $76.92 \%$ \\
\hline Max & 140.0 & 1051 & 7.5 & 117.0 & 9.4 & 61 & 20.2 & 138 & 1275 & 11.3 & 110 & 14.4 & $104.3 \%$ & $103.9 \%$ & $13.1 \%$ & $91.74 \%$ & $50.41 \%$ & $86.54 \%$ \\
\hline Fict-0774-01 & 359 & 28,252 & 78.7 & 318 & 88.8 & 93 & 303.8 & 306 & 17,528 & 57.3 & 267 & 65.6 & $-27.2 \%$ & $-26.1 \%$ & $-78.4 \%$ & $88.58 \%$ & $25.91 \%$ & $87.25 \%$ \\
\hline Fict-0774-02 & 276 & 11,684 & 42.3 & 265 & 44.1 & 101 & 115.7 & 311 & 13,664 & 43.9 & 301 & 45.4 & $3.8 \%$ & $3.0 \%$ & $-60.8 \%$ & $96.01 \%$ & $36.59 \%$ & $96.78 \%$ \\
\hline Fict-0774-03 & 245 & 13,906 & 56.8 & 237 & 58.7 & 102 & 136.3 & 287 & 13,374 & 46.6 & 275 & 48.6 & $-17.9 \%$ & $-17.1 \%$ & $-64.3 \%$ & $96.73 \%$ & $41.63 \%$ & $95.82 \%$ \\
\hline Fict-0774-04 & 292 & 8254 & 28.3 & 265 & 31.1 & 99 & 83.4 & 335 & 6720 & 20.1 & 310 & 21.7 & $-29.0 \%$ & $-30.4 \%$ & $-74.0 \%$ & $90.75 \%$ & $33.90 \%$ & $92.54 \%$ \\
\hline Fict-0774-05 & 279 & 11,348 & 40.7 & 266 & 42.7 & 104 & 109.1 & 304 & 7702 & 25.3 & 296 & 26.0 & $-37.7 \%$ & $-39.0 \%$ & $-76.2 \%$ & $95.34 \%$ & $37.28 \%$ & $97.37 \%$ \\
\hline Fict-0774-08 & 311 & 10,999 & 35.4 & 293 & 37.5 & 104 & 105.8 & 381 & 12,315 & 32.3 & 351 & 35.1 & $-8.6 \%$ & $-6.5 \%$ & $-66.8 \%$ & $94.21 \%$ & $33.44 \%$ & $92.13 \%$ \\
\hline Fict-0774-09 & 257 & 15,049 & 58.6 & 253 & 59.5 & 102 & 147.5 & 290 & 14,963 & 51.6 & 280 & 53.4 & $-11.9 \%$ & $-10.2 \%$ & $-63.8 \%$ & $98.44 \%$ & $39.69 \%$ & $96.55 \%$ \\
\hline Fict-0774-10 & 313 & 31,287 & 100.0 & 308 & 101.6 & 129 & 242.5 & 348 & 44,381 & 127.5 & 343 & 129.4 & $27.6 \%$ & $27.4 \%$ & $-46.6 \%$ & $98.40 \%$ & $41.21 \%$ & $98.56 \%$ \\
\hline Avg. & 291.2 & 17,742 & 60.2 & 275.6 & 63.4 & 103 & 171.8 & 323.1 & 22,712 & 68.6 & 306.7 & 71.7 & $3.8 \%$ & $3.6 \%$ & $-61.1 \%$ & $94.86 \%$ & $35.79 \%$ & $94.94 \%$ \\
\hline Min & 245.0 & 8254 & 28.3 & 237.0 & 31.1 & 93 & 83.4 & 287 & 6720 & 20.1 & 267 & 21.7 & $-37.7 \%$ & $-39.0 \%$ & $-78.4 \%$ & $88.58 \%$ & $25.91 \%$ & $87.25 \%$ \\
\hline $\operatorname{Max}$ & 359.0 & 31,287 & 100.0 & 318.0 & 101.6 & 129 & 303.8 & 381 & 68,899 & 193.5 & 351 & 198.6 & $116.6 \%$ & $114.2 \%$ & $-24.9 \%$ & $98.44 \%$ & $41.63 \%$ & $98.56 \%$ \\
\hline
\end{tabular}




\section{Conclusions}

This paper studies the Bi-Objective Insular Traveling Salesman Problem (BO-InTSP), which minimizes both the maritime transportation costs (MTC) incurred by a barge that visits a set of islands for freight collection (or distribution) purposes, and the ground transportation costs (GTC) incurred by the inhabitants inside the islands when moving the freight. A first formulation is proposed in [39], which relies on an approximation of the GTC that assumes the existence of a set of centroids instead of using the actual demand locations. Naturally, the use of an approximated GTC may lead to an inappropriate set of solutions, manifesting the need for studying the quality of this approximation. Accordingly, this paper proposes a novel Exact Formulation for the studied problem by modelling the actual demand locations and an explicit assignment of these locations to the visited ports at each island. Subsequently, this research proposes and develops a systematic evaluation approach for comparing the results obtained with both the Exact and the Approximated Formulations, in which the bi-objective nature of the problem is taken into account. A key step of the comparison approach is the exact re-computation of the GTC for each point that is obtained with the Approximation Model. In other words, the Pareto Front obtained with the Approximated Model is projected into the objective space of the Exact Formulation.

Commonly, traditional multi-objective approaches compare the sets of non-dominated points generated by different approximated algorithms (i.e., heuristic) for a single problem or model formulation. In this research, we propose an enhanced analysis that compares between models with different accuracy or aggregation levels, which may be relevant when trying to balance the effort needed to solve a problem either through an Exact Formulation or through an Approximated Model.

The results show significant differences when the set of non-dominated points obtained by the proposed Exact Formulation is compared with those points obtained by the previously studied Approximated Model. First, when the GTC is exactly recomputed for each point obtained by the Approximated Model, many of these solutions are not actually efficient and must be removed from the set, representing approximately $56 \%$ of the initial set obtained for the tested instances (51\% for the real instances, and $62 \%$ for the fictitious instances). Additionally, approximately $40 \%$ of the points from the Approximated Pareto Front actually belong to the Exact Pareto Front (51\% for the real instances and 29\% for the fictitious ones), representing approximately $20 \%$ of the Exact Pareto Front (29\% for the real instances and $10 \%$ for the fictitious ones).

The proposed novel Exact Formulation for the BO-InTSP yields significantly larger sets of non-dominated points in similar computing times when compared to the previous Approximated Model in [39], which provides an Approximated Pareto Set whose cardinality is approximately $45 \%$ of the Exact Pareto Set. Thus, the novel proposed Exact Formulation clearly outperforms the Approximated Model, at least for the set of instances explored in this research. Nevertheless, the quality of the set of points obtained with the previous Approximated Model is acceptable considering the high values of its dominated areas and the low error values of its dominated solutions. Notice that this comparison cannot be performed without the proposed Exact Formulation.

Based on this research, it is worth highlighting the great relevance of the modelling approach for the GTC (in addition to multi-objective solution approaches in order to solve the underlying optimization models), since it significantly affects the quality of the obtained Pareto Front and also the consumed computing resources.

In this paper, the performance of different Pareto Fronts has been compared by developing a systematic approach that relies on a variety of state-of-the-art performance indicators. In this regard, well-known multi-objective methodologies are extended for comparing the Pareto Sets obtained with both Exact and Approximated Formulations for a given optimization problem. Thus, this paper proposes a systematic approach to provide a fair and thorough comparison between the respective sets. Moreover, this approach 
may be naturally extended to problems with three or more objective functions, denoting a significant contribution of this research.

Alternative approximations or modelling techniques for the GTC inside the islands, such as probabilistic and gravitational-based models, are suggested as a relevant future research. In particular, a stochastic approach should be adopted in case of real demand locations that are of a random nature. Naturally, considering the bi-objective and NPhard nature of the studied problem, multi-objective heuristics techniques for solving both formulations are highlighted as a significant and necessary future research to be performed, particularly when larger instances are intended to be solved (e.g., MOEA/D, NSGA-II, and Pareto Local Search, and all of their variations and improvements [75-80]). Finally, some interesting extensions may be addressed, such as multi-period, multi-vehicle, and multi-depot scenarios.

Author Contributions: Conceptualization, P.A.M.-G., J.M.-R., C.A.B. and G.C.-G.; data curation, P.A.M.-G. and J.M.-R.; formal analysis, P.A.M.-G. and J.M.-R.; funding acquisition, P.A.M.-G.; investigation, P.A.M.-G., J.M.-R., C.A.B. and G.C.-G.; methodology, P.A.M.-G., J.M.-R., C.A.B. and G.C.-G.; project administration, P.A.M.-G.; resources, P.A.M.-G.; software, J.M.-R.; supervision, P.A.M.-G.; validation, P.A.M.-G., J.M.-R., C.A.B. and G.C.-G.; visualization, P.A.M.-G. and J.M.-R.; writing-original draft, P.A.M.-G. and C.A.B.; writing-review and editing, P.A.M.-G., J.M.-R., C.A.B. and G.C.-G. All authors have read and agreed to the published version of the manuscript.

Funding: This research received no external funding.

Institutional Review Board Statement: Not applicable.

Informed Consent Statement: Not applicable.

Data Availability Statement: Not applicable.

Conflicts of Interest: The authors declare no conflict of interest. The funders had no role in the design of the study; in the collection, analyses, or interpretation of data; in the writing of the manuscript, or in the decision to publish the results.

\section{Appendix A. Pareto Fronts for Real-Based Instances}

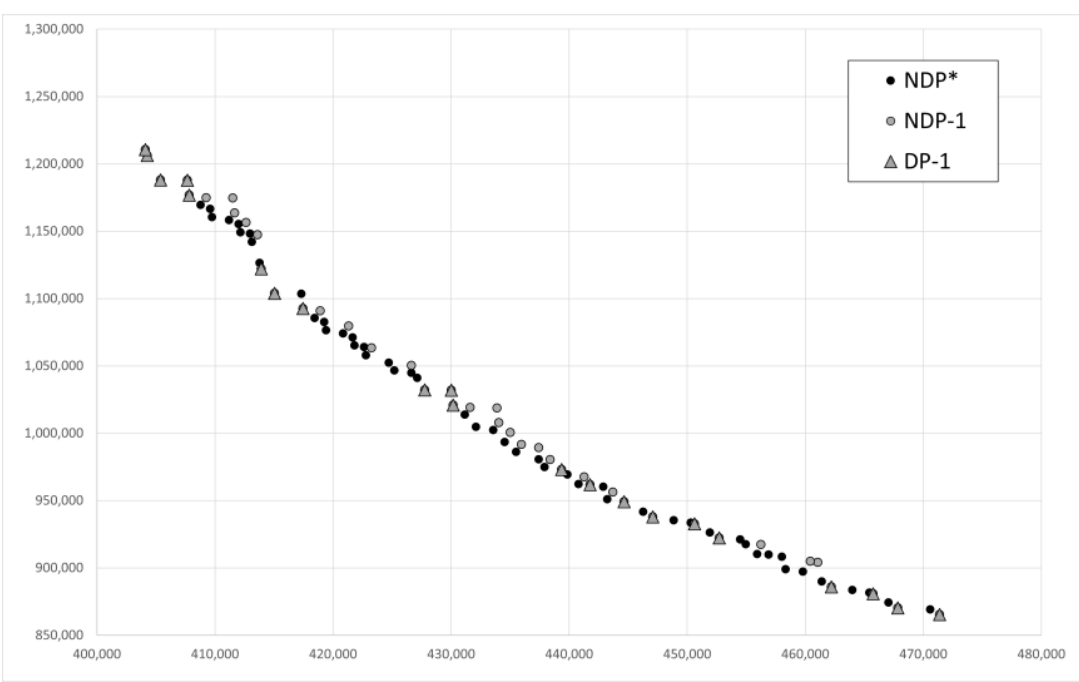

Figure A1. Pareto Fronts for instance Real-8820-01. 


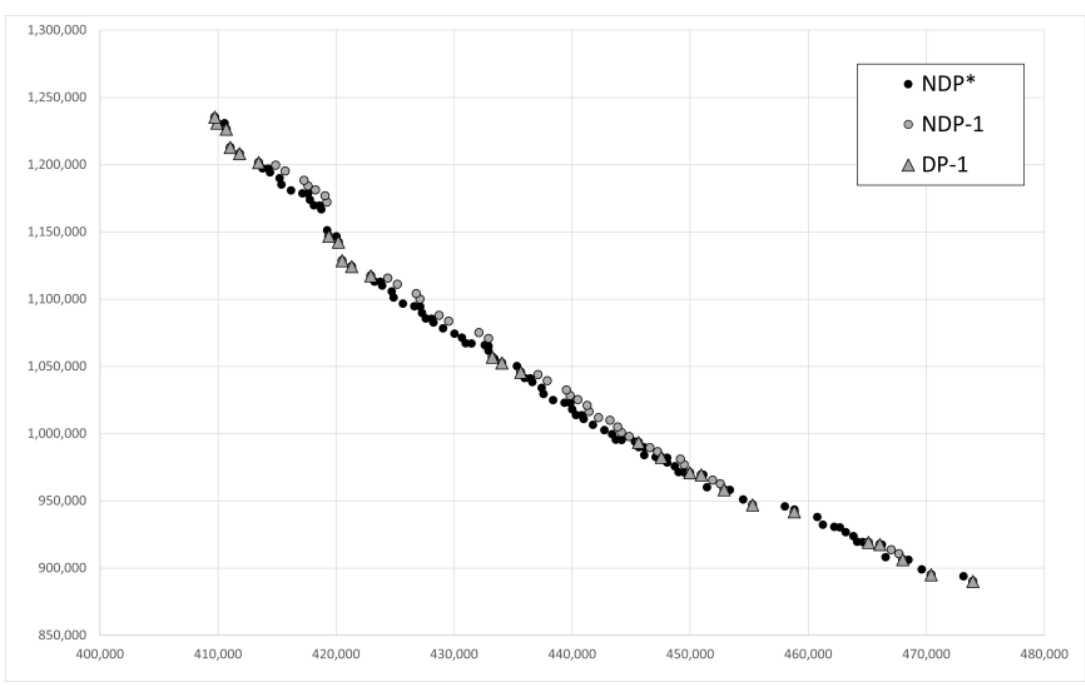

Figure A2. Pareto Fronts for instance Real-8820-02.

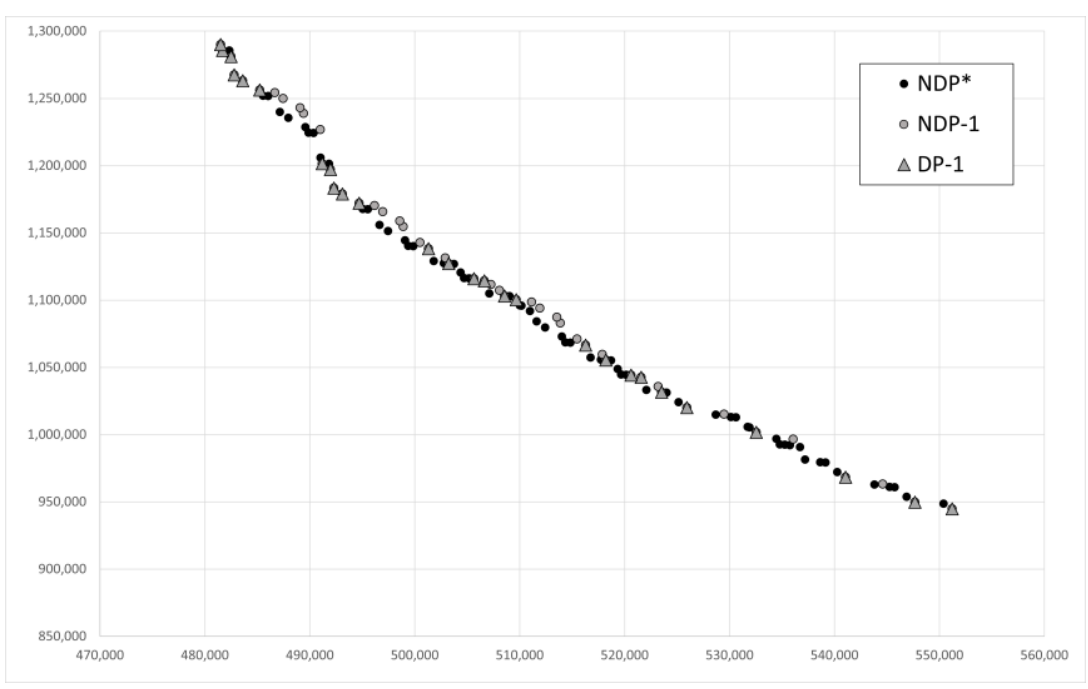

Figure A3. Pareto Fronts for instance Real-8820-03.

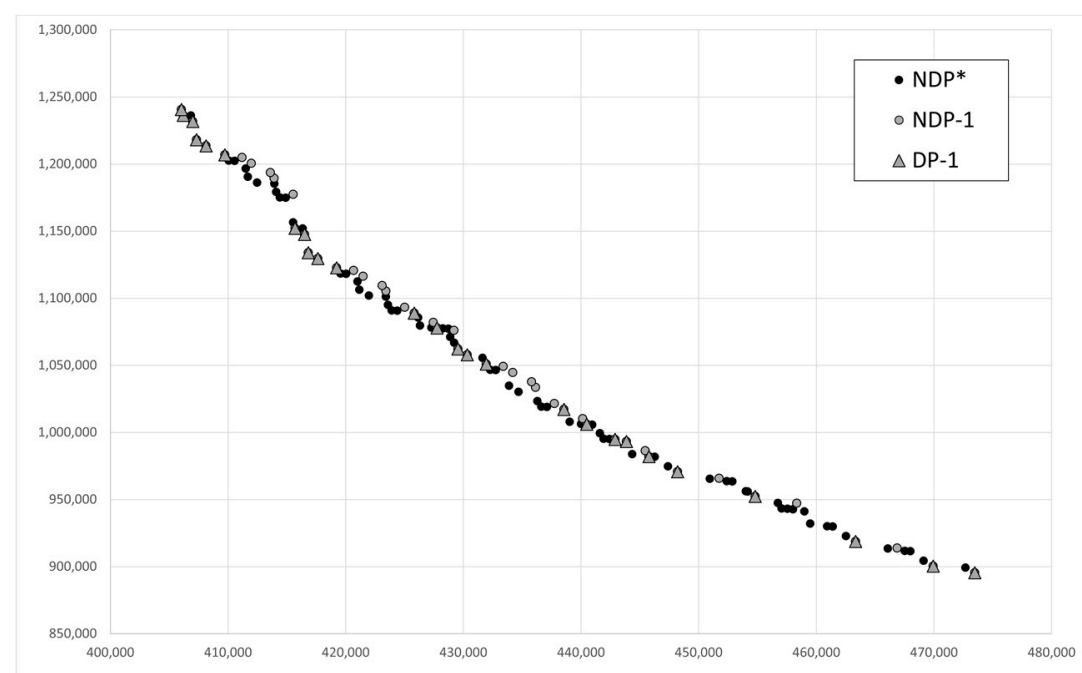

Figure A4. Pareto Fronts for instance Real-8820-04. 


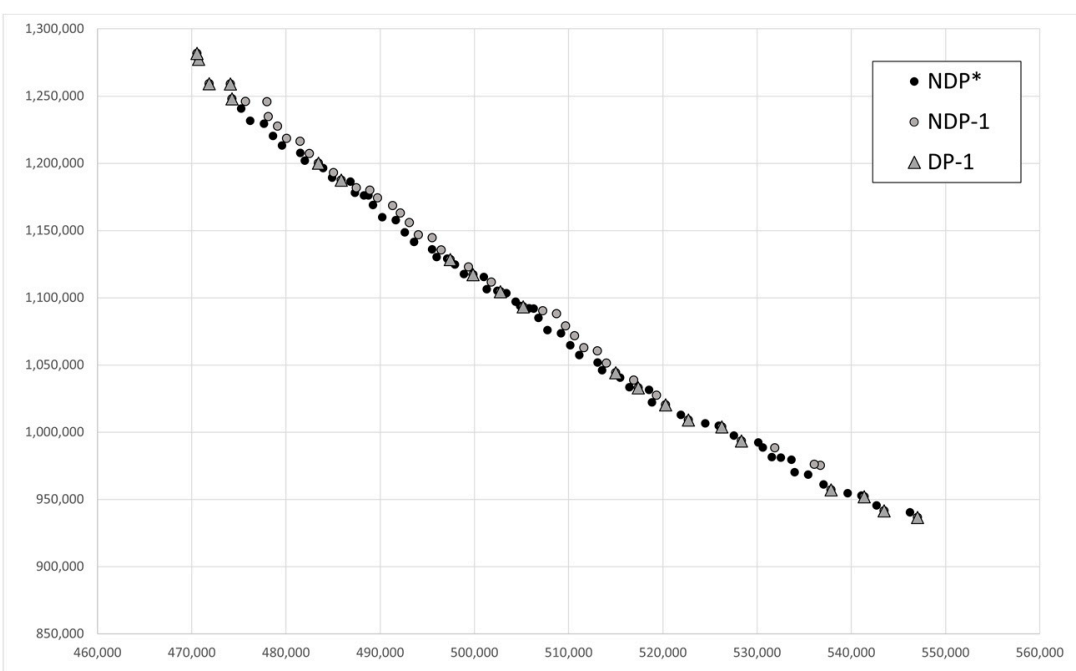

Figure A5. Pareto Fronts for instance Real-8820-05.

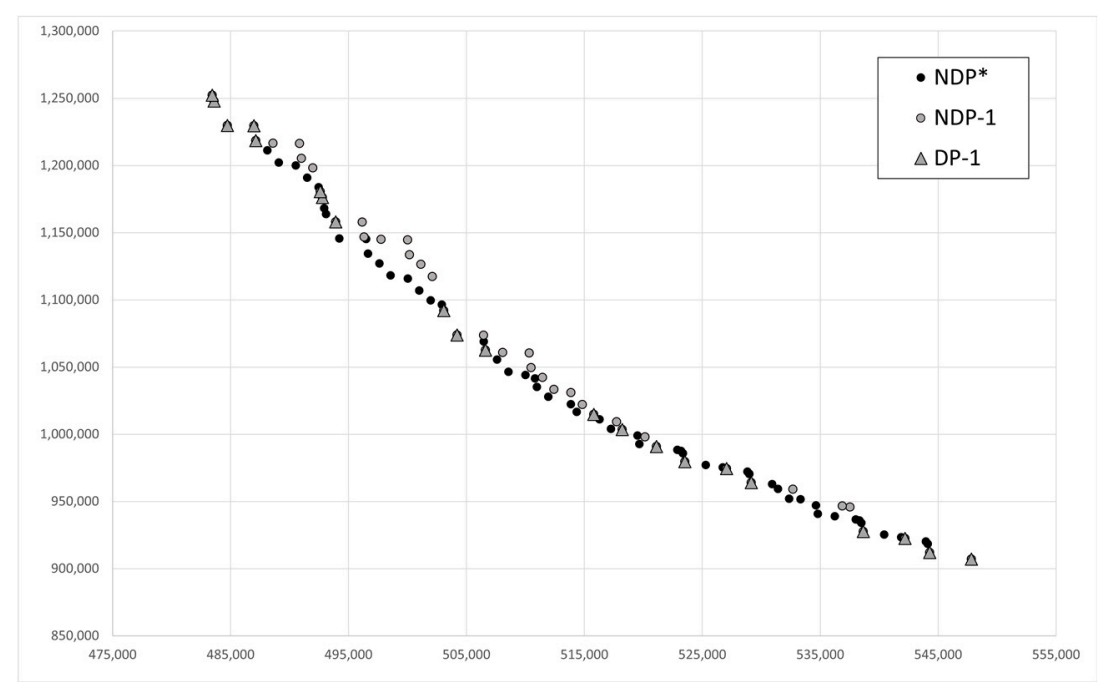

Figure A6. Pareto Fronts for instance Real-8820-06.

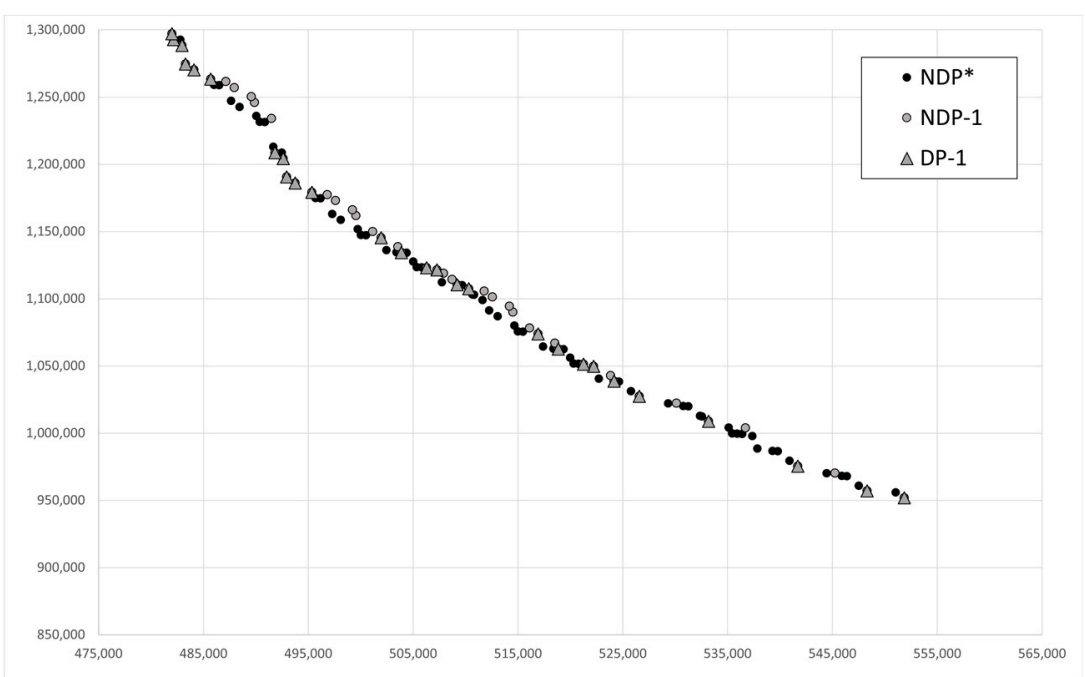

Figure A7. Pareto Fronts for instance Real-8820-07. 


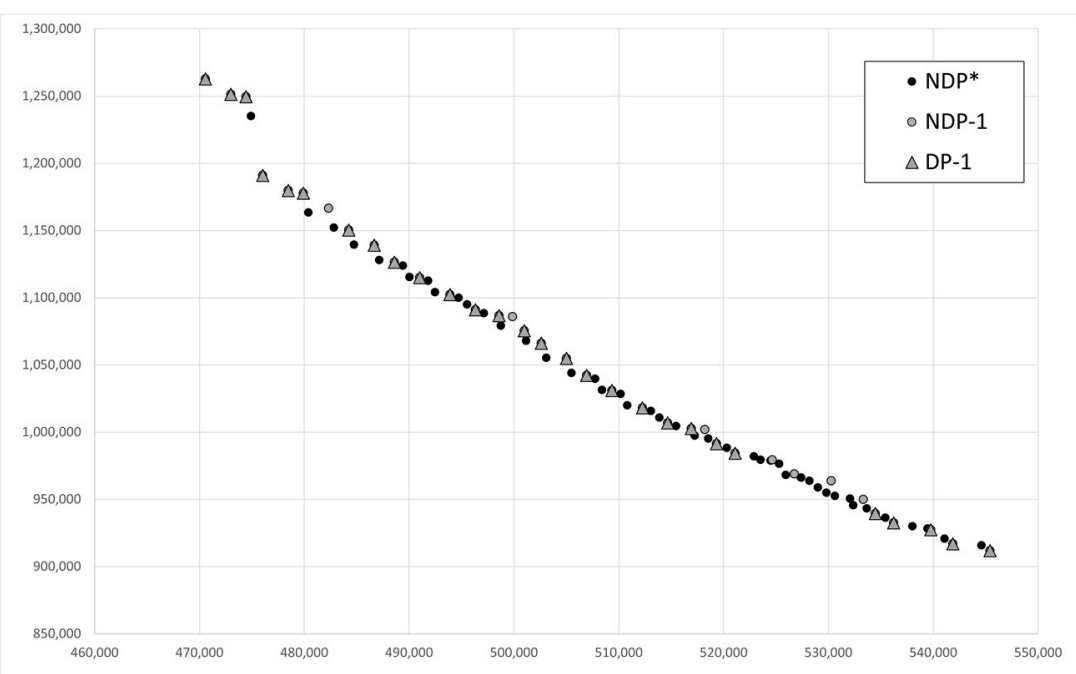

Figure A8. Pareto Fronts for instance Real-8820-08.

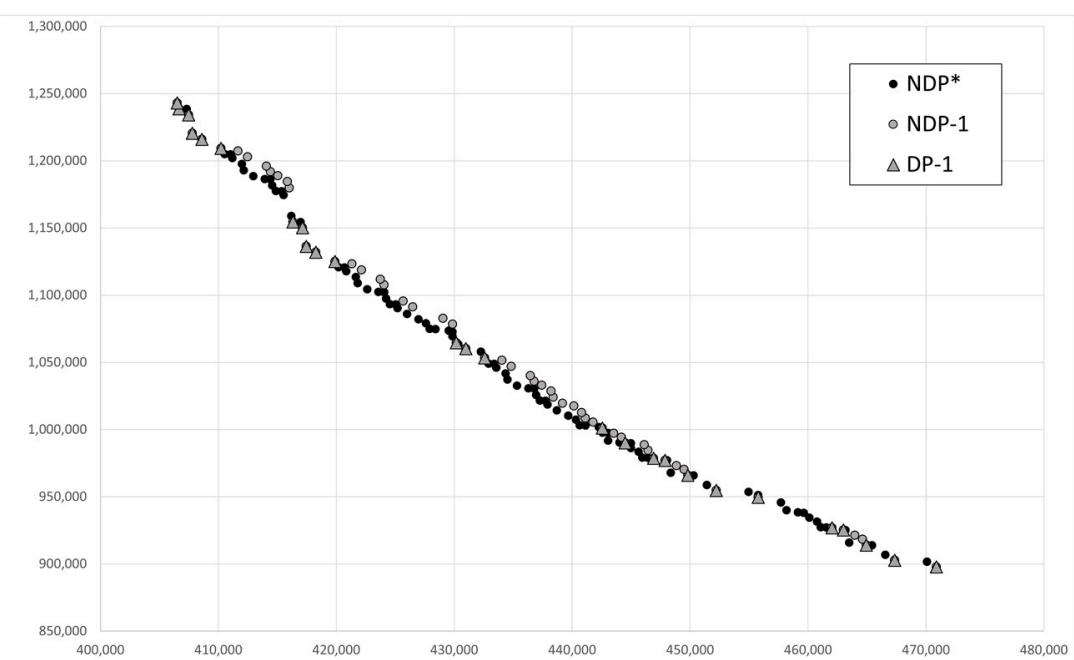

Figure A9. Pareto Fronts for instance Real-8820-09.

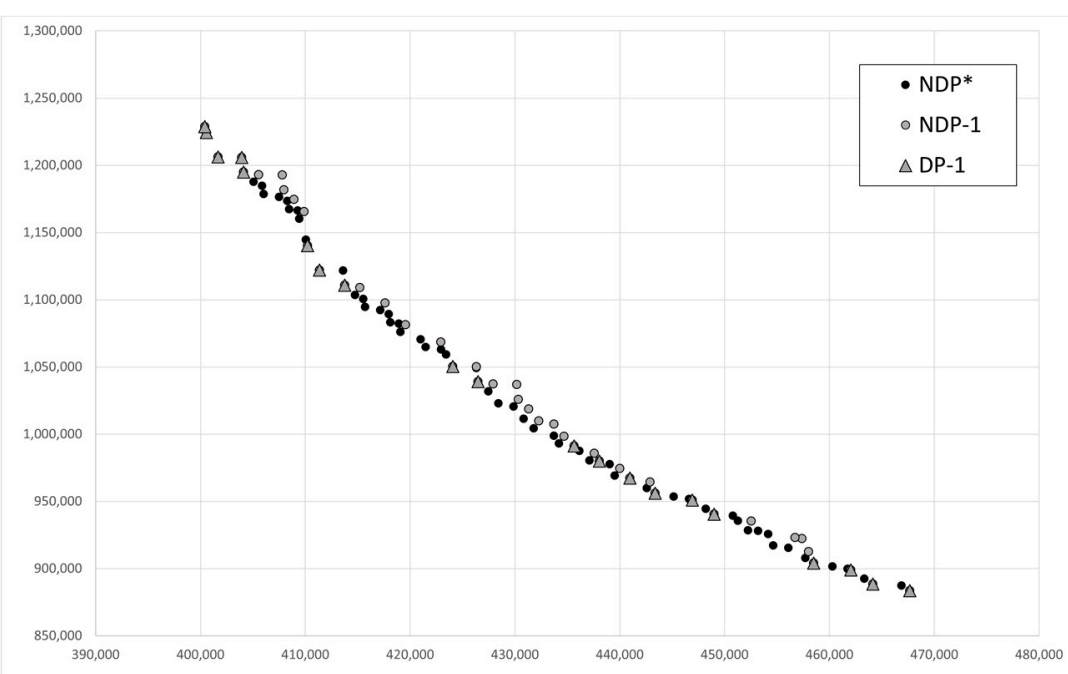

Figure A10. Pareto Fronts for instance Real-8820-10. 


\section{References}

1. Laporte, G.; Osman, I.H. Routing problems: A bibliography. Ann. Oper. Res. 1995, 61, 227-262. [CrossRef]

2. Laporte, G.; Gendreau, M.; Potvin, J.-Y.; Semet, F. Classical and modern heuristics for the vehicle routing problem. Int. Trans. Oper. Res. 2000, 7, 285-300. [CrossRef]

3. Ando, N.; Taniguchi, E. Travel time reliability in vehicle routing and scheduling with time windows. Netw. Spat. Econ. 2006, 6, 293-311. [CrossRef]

4. Eksioglu, B.; Vural, A.V.; Reisman, A. The vehicle routing problem: A taxonomic review. Comput. Ind. Eng. 2009, 57, 1472-1483. [CrossRef]

5. Pillac, V.; Gendreau, M.; Guéret, C.; Medaglia, A.L. A review of dynamic vehicle routing problems. Eur. J. Oper. Res. 2013, 225, 1-11. [CrossRef]

6. Braekers, K.; Ramaekers, K.; Van Nieuwenhuyse, I. The vehicle routing problem: State of the art classification and review. Comput. Ind. Eng. 2016, 99, 300-313. [CrossRef]

7. Laporte, G.; Martello, S. The Selective Travelling Salesman Problem. Discret. Appl. Math. 1990, 26, 193-207. [CrossRef]

8. Gendreau, M.; Laporte, G.; Semet, F. A branch-and-cut algorithm for the undirected selective traveling salesman problem. Networks 1998, 32, 263-273. [CrossRef]

9. Gutiérrez-Jarpa, G.; Marianov, V.; Obreque, C. A single vehicle routing problem with fixed delivery and optional collections. IIE Trans. 2009, 41, 1067-1079. [CrossRef]

10. Sabo, C.; Pop, P.C.; Horvat-Marc, A. On the selective vehicle routing problem. Mathematics 2020, 8, 771. [CrossRef]

11. Golden, B.L.; Levy, L.; Vohra, R. The orienteering problem. Nav. Res. Logist. 1987, 34, 307-318. [CrossRef]

12. Chao, I.-M.; Golden, B.L.; Wasil, E.A. The team orienteering problem. Eur. J. Oper. Res. 1996, 88, 464-474. [CrossRef]

13. Fischetti, M.; Salazar, J.; Toth, P. Solving the orienteering problem through branch-and-cut. INFORMS J. Comput. 1998, 10, 133-148. [CrossRef]

14. Vansteenwegen, P.; Souffriau, W.; Oudheusden, D. The orienteering problem: A survey. Eur. J. Oper. Res. 2001, 209, 1-10. [CrossRef]

15. Archetti, C.; Bianchessi, N.; Speranza, M.G. Optimal solutions for routing problems with profits. Discret. Appl. Math. 2013, 161, 547-557. [CrossRef]

16. Bianchessi, N.; Mansini, R.; Speranza, M.G. A branch-and-cut algorithm for the Team Orienteering Problem. Int. Trans. Oper. Res. 2018, 25, 627-635. [CrossRef]

17. Estrada-Moreno, F.A.; Juan, A.A.; Panadero, J.; Bagirov, A. The non-smooth and bi-objective team orienteering problem with soft constraints. Mathematics 2020, 8, 1461. [CrossRef]

18. Laporte, G.; Asef-Vaziri, A.; Sriskandarajah, C. Some applications of the generalized travelling salesman problem. J. Oper. Res. Soc. 1996, 47, 1461-1467. [CrossRef]

19. Pop, P.C. New integer programming formulations of the generalized traveling salesman problem. Am. J. Appl. Sci. 2007, 4, 932-937.

20. Pop, P.C. Generalized Network Design Problems. De Gruyter Ser. Discret. Math. Appl. 2012, 15, 7686.

21. Karapetyan, D.; Gutin, G. Efficient local search algorithms for known and new neighborhoods for the generalized traveling salesman problem. Eur. J. Oper. Res. 2012, 219, 234-251. [CrossRef]

22. Pop, P.C.; Kara, I.; Marc, A.H. New mathematical models of the generalized vehicle routing problem and extensions. Appl. Math Model. 2012, 36, 97-107. [CrossRef]

23. Naji-Azimi, Z.; Salari, M. The time constrained maximal covering salesman problem. Appl. Math. Model. 2014, 38, 3945-3957. [CrossRef]

24. Afsar, H.M.; Prins, C.; Santos, A.C. Exact and heuristic algorithms for solving the generalized vehicle routing problem with flexible fleet size. Int. Trans. Oper. Res. 2014, 21, 153-175. [CrossRef]

25. Ardalan, Z.; Karimi, S.; Poursabzi, O.; Naderi, B. A novel imperialist competitive algorithm for generalized traveling salesman problems. Appl. Soft Comput. 2015, 26, 546-555. [CrossRef]

26. Obreque, C.; Paredes-Belmar, G.; Miranda, P.; Campusano, G.; Gutierrez-Jarpa, G. The Generalized Median Tour Problem: Modeling, Solving and an Application. IEEE Access 2020, 8, 178097-178107. [CrossRef]

27. Pop, P.C.; Matei, O.; Sitar, C.P. An improved hybrid algorithm for solving the generalized vehicle routing problem. Neurocomputing 2013, 109, 76-83. [CrossRef]

28. Mesa, J.A.; Boffey, T.B. A review of extensive facility location in networks. Eur. J. Oper. Res. 1996, 95, 592-603. [CrossRef]

29. Labbé, M.; Laporte, G.; Rodríguez-Martín, I. Path, tree and cycle location. In Fleet Management and Logistics; Springer: Berlin/Heidelberg, Germany, 1998. [CrossRef]

30. Contreras, I.; Fernández, E. General network design: A unified view of combined location and network design problems. Eur. J. Oper. Res. 2012, 219, 680-697. [CrossRef]

31. Pop, P.C.; Sitar, C.P. New models of the generalized fixed-charge network design problem. Carpathian J. Math. 2012, 28, 43-150. [CrossRef]

32. Clímaco, J.C.N.; Pascoal, M. Multicriteria path and tree problems: Discussion on exact algorithms and applications. Int. Trans. Oper. Res. 2012, 19, 63-98. [CrossRef] 
33. Puerto, J.; Ricca, F.; Scozzari, A. Extensive facility location problems on networks: An updated review. TOP 2018, 26, 187-226. [CrossRef]

34. Mesa, J. Comments on: Extensive facility location problems on networks: An updated review. TOP 2018, 26, 227-228. [CrossRef]

35. Saldanha-da-Gama, F. Comments on: Extensive facility location problems on networks: An updated review. TOP 2018, 26, 229-232. [CrossRef]

36. Pop, P.C.; Matei, O.; Saboa, C.; Petrovan, A. A two-level solution approach for solving the generalized minimum spanning tree problem. Eur. J. Oper. Res. 2018, 265, 478-487. [CrossRef]

37. Miranda, P.; Blazquez, C.; Vergara, R.; Weitzler, S. A novel methodology for designing a household waste collection system for insular zones. Transportation Research Part E 2015, 77, 227-247. [CrossRef]

38. Arango Gonzalez, D.S.; Olivares-Benitez, E.; Miranda, P.A. Insular Biobjective Routing with Environmental Considerations for a Solid Waste Collection System in Southern Chile. Adv. Oper. Res. 2017, 11, 4093689. [CrossRef]

39. Miranda, P.A.; Blazquez, C.A.; Obreque, C.; Maturana-Ross, J.; Gutierrez-Jarpa, G. The bi-objective insular traveling salesman problem with maritime and ground transportation costs. Eur. J. Oper. Res. 2018, 271, 1014-1036. [CrossRef]

40. Cummins, S.; MacIntyre, S. Food deserts: Evidence and assumption in health policy making. Br. Med J. 2002, 325, 436-438. [CrossRef]

41. Cummins, S.; MacIntyre, S. Systematic study of an urban foodscape: The price and availability of food in Greater Glasgow. Urban Stud. 2002, 39, 2115-2130. [CrossRef]

42. Guy, C.; David, G. Measuring geographical access to 'healthy foods' in areas of social deprivation: A case study in Cardiff. Int. J. Consum. Stud. 2004, 28, 222-224. [CrossRef]

43. Apparicio, P.; Cloutier, M.; Shearmur, R. The case of Montréal's missing food deserts: Evaluation of accessibility to food supermarkets. Int. J. Health Geogr. 2007, 6, 1476. [CrossRef]

44. Caramaschi, S. Counteracting Food Deserts: The potential for mobile food vending in regenerating contemporary cities. Int. J. Sustain. Dev. Plan. 2017, 12, 744-751. [CrossRef]

45. Dal Fior, G. An Analytical Framework to explore the phenomenon of Food Deserts: A case of Portsmouth. In Master in Ingegneria Gestionale; Università degli Studi di Padova-Dipartimento di Tecnica e Gestione dei Sistemi Industriali: Vicenza, Italy, 2019.

46. Irawan, C.A.; Jones, D.; Ouelhadj, D. Bi-objective optimisation model for installation scheduling in offshore wind farms. Comput. Oper. Res. 2017, 78, 393-407. [CrossRef]

47. Irawan, C.A.; Ouelhadj, D.; Jones, D.; Stålhane, M.; Sperstad, I.B. Optimisation of maintenance routing and scheduling for offshore wind farms. Comput. Oper. Res. 2017, 256, 76-89. [CrossRef]

48. Chisman, J.A. The clustered traveling salesman problem. Comput. Oper. Res. 1975, 2, 115-119. [CrossRef]

49. Battarra, M.; Erdoğan, G.; Vigo, D. Exact algorithms for the clustered vehicle routing problem. Oper. Res. 2014, 62, 58-71. [CrossRef]

50. Vidal, T.; Battarra, M.; Subramanian, A.; Erdoğan, G. Hybrid metaheuristics for the Clustered Vehicle Routing Problem. Comput. Oper. Res. 2015, 58, 87-99. [CrossRef]

51. Jozefowiez, N.; Semet, F.; Talbi, E.-G. Multi-objective vehicle routing problems. Eur. J. Oper. Res. 2008, 189, 293-309. [CrossRef]

52. Wang, J.; Zhou, Y.; Wang, Y.; Zhang, J.; Chen, C.L.P.; Zheng, Z. Multiobjective Vehicle Routing Problems with Simultaneous Delivery and Pickup and Time Windows: Formulation, Instances, and Algorithms. IEEE Trans. Cybern. 2016, 46, 582-594. [CrossRef]

53. Lust, T.; Teghem, J. The multiobjective traveling salesman problem: A survey and a new approach. Stud. Comput. Intell. 2010, 272, 119-141.

54. Guerriero, F.; De Rango, F.; Marano, S.; Bruno, E. A biobjective optimization model for routing in mobile ad hoc networks. Appl. Math. Model. 2009, 33, 1493-1512. [CrossRef]

55. Jabali, O.; Van Woensel, T.; De Kok, A.G. Analysis of travel times and $\mathrm{CO}_{2}$ emissions in time-dependent vehicle routing. Prod. Oper. Manag. 2012, 21, 1060-1074. [CrossRef]

56. Paquete, L.; Jaschob, M.; Klamroth, K.; Gorski, J. On a biobjective search problem in a line: Formulations and algorithms. Theor. Comput. Sci. 2013, 507, 61-71. [CrossRef]

57. Rodríguez-Zbinden, S.; Montero, C.; Blázquez Miranda, P. A Multi-objective Iterated Local Search Approach to Solve the Insular Traveling Salesman Problem. Congr. Evol. Comput. 2021, 48, 2339-2346.

58. Farmani, R.; Savic, D.A.; Walters, G.A. Evolutionary multi-objective optimization in water distribution network design. Eng. Optim. 2005, 37, 167-183. [CrossRef]

59. Wang, F.; Lai, X.; Shi, N. A multi-objective optimization for green supply chain network design. Decis. Support Syst. 2011, 51, 262-269. [CrossRef]

60. Arbex, R.O.; da Cunha, C.B. Efficient transit network design and frequencies setting multi-objective optimization by alternating objective genetic algorithm. Transp. Res. Part B 2015, 81, 355-376. [CrossRef]

61. Current, J.R.; Schilling, D.A. The median tour and maximal covering tour problems: Formulations and heuristics. Eur. J. Oper. Res. 1994, 73, 114-126. [CrossRef]

62. Current, J.R.; Revelle, C.S.; Cohon, J.L. The median shortest path problem: A multiobjective approach to analyze cost vs. accessibility in the design of transportation networks. Transp. Sci. 1987, 21, 188-197. [CrossRef] 
63. Liefooghe, A.; Jourdan, L.; Talbi, E.-G. Metaheuristics and cooperative approaches for the Bi-objective Ring Star Problem. Comput. Oper. Res. 2010, 37, 1033-1044. [CrossRef]

64. Calvete, H.I.; Galé, C.; Iranzo, J.A. MEALS: A multi-objective evolutionary algorithm with local search for solving the bi-objective ring star problem. Eur. J. Oper. Res. 2016, 250, 377-388. [CrossRef]

65. Jozefowiez, N.; Semet, F.; Talbi, E.-G. The bi-objective covering tour problem. Comput. Oper. Res. 2007, 34, 1929-1942. [CrossRef]

66. Riera-Ledesma, J.; Salazar-González, J.J. The biobjective travelling purchaser problem. Eur. J. Oper. Res. 2005, 160, 599-613. [CrossRef]

67. Manerba, D.; Mansini, R.; Riera-Ledesma, J. The Traveling Purchaser Problem and its variants. Eur. J. Oper. Res. 2017, 259 , 1-18. [CrossRef]

68. Zitzler, E.; Thiele, L. Multiobjective evolutionary algorithms: A comparative case study and the strength Pareto approach. IEEE Trans. Evol. Comput. 1999, 3, 257-271. [CrossRef]

69. Peng, W.; Zhang, Q.; Li, H. Comparison between MOEA/D and NSGA-II on the Multi-Objective Travelling Salesman Problem. Stud. Comput. Intell. 2009, 171, 309-324.

70. Chiandussi, G.; Codegoneb, M.; Ferrero, S.; Varesio, F.E. Comparison of multi-objective optimization methodologies for engineering applications. Comput. Math. Appl. 2012, 63, 912-942. [CrossRef]

71. Psychas, I.-D.; Delimpasi, E.; Marinakis, Y. Hybrid evolutionary algorithms for the Multiobjective Traveling Salesman Problem. Expert Syst. Appl. 2015, 42, 8956-8970. [CrossRef]

72. Mavrotas, G.; Florios, K. An improved version of the augmented $\varepsilon$-constraint method (AUGMECON2) for finding the exact pareto set in multi-objective integer programming problems. Appl. Math. Comput. 2013, 219, 9652-9669. [CrossRef]

73. Mavrotas, G. Effective implementation of the e-constraint method in multi-objective mathematical programming problems. Appl. Math. Comput. 2009, 213, 455-465. [CrossRef]

74. Gavish, B.; Graves, S. The Travelling Salesman Problem and Related Problems; Working Paper OR-078-78; Operations Research Center, MIT: Cambridge, MA, USA, 1978.

75. Deb, K.; Pratap, A.; Agarwal, S.; Meyarivan, T. A fast and elitist multiobjective genetic algorithm: NSGA-II. IEEE Trans. Evol. Comput. 2002, 6, 182-197. [CrossRef]

76. Li, H.; Zhang, Q. Multiobjective optimization problems with complicated pareto sets, MOEA/D and NSGA-II. IEEE Trans. Evol. Comput. 2009, 13, 284-302. [CrossRef]

77. Lust, T.; Teghem, J. Two-phase pareto local search for the biobjective traveling salesman problem. J. Heuristics 2010, 16, 475-510. [CrossRef]

78. Zhang, Q.; Li, H. MOEA/D: A multiobjective evolutionary algorithm based on decomposition. IEEE Trans. Evol. Comput. 2007, 11, 712-731. [CrossRef]

79. Dubois-Lacoste, J.; López-Ibáñez, M.; Stützle, T. Anytime Pareto local search. Eur. J. Oper. Res. 2015, 243, 369-385. [CrossRef]

80. Peng, W.; Zhang, Q.; Li, H. Comparison between MOEA/D and NSGA-II on the Multi-Objective Travelling Salesman Problem. In Multi-Objective Memetic Algorithms; Studies in Computational Intelligence; Springer: Berlin/Heidelberg, Germany, 2009; Volume 171. [CrossRef]

81. Zitzler, E.; Deb, K.; Thiele, L. Comparison of multiobjective evolutionary algorithms: Empirical results. Evol. Comput. 2000, 8, 173-195. [CrossRef]

82. Zitzler, E.; Thiele, L.; Laumanns, M.; Fonseca, C.M.; da Fonseca, V.G. Performance assessment of multiobjective optimizers: An analysis and review. IEEE Trans. Evol. Comput. 2003, 7, 117-132. [CrossRef] 